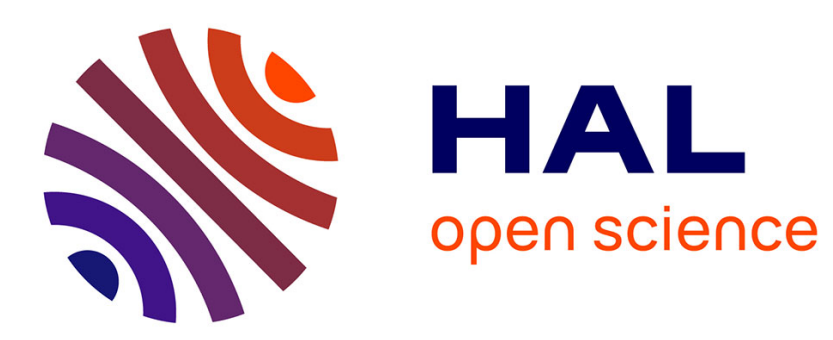

\title{
Influence of nozzle-exit boundary-layer conditions on the flow and acoustic fields of initially laminar jets
}

\author{
Christophe Bogey, Christophe Bailly
}

\section{To cite this version:}

Christophe Bogey, Christophe Bailly. Influence of nozzle-exit boundary-layer conditions on the flow and acoustic fields of initially laminar jets. Journal of Fluid Mechanics, 2010, 663, pp.507-538. 10.1017/S0022112010003605. hal-00566006

\section{HAL Id: hal-00566006 https://hal.science/hal-00566006}

Submitted on 11 Jun 2012

HAL is a multi-disciplinary open access archive for the deposit and dissemination of scientific research documents, whether they are published or not. The documents may come from teaching and research institutions in France or abroad, or from public or private research centers.
L'archive ouverte pluridisciplinaire HAL, est destinée au dépôt et à la diffusion de documents scientifiques de niveau recherche, publiés ou non, émanant des établissements d'enseignement et de recherche français ou étrangers, des laboratoires publics ou privés. 


\title{
Influence of nozzle-exit boundary-layer conditions on the flow and acoustic fields of initially laminar jets
}

\author{
C. BOGEY $Y^{1} \dagger$ AND C. BAILL $Y^{1,2}$ \\ ${ }^{1}$ Laboratoire de Mécanique des Fluides et d'Acoustique, UMR CNRS 5509, \\ Ecole Centrale de Lyon, 69134 Ecully CEDEX, France \\ ${ }^{2}$ Institut Universitaire de France, 103 Boulevard Saint-Michel, 75005 Paris, France
}

(Received 16 October 2009; revised 5 July 2010; accepted 5 July 2010)

Round jets originating from a pipe nozzle are computed by large-eddy simulations (LES) to investigate the effects of the nozzle-exit conditions on the flow and sound fields of initially laminar jets. The jets are at Mach number 0.9 and Reynolds number $10^{5}$, and exhibit exit boundary layers characterized by Blasius velocity profiles, maximum root-mean-square (r.m.s.) axial velocity fluctuations between 0.2 and $1.9 \%$ of the jet velocity, and momentum thicknesses varying from 0.003 to 0.023 times the jet radius. The far-field noise is determined from the LES data on a cylindrical surface by solving the acoustic equations. Jets with a thinner boundary layer develop earlier but at a slower rate, yielding longer potential cores and lower centreline turbulent intensities. Adding random pressure disturbances of low magnitude in the nozzle also increases the potential core length and reduces peak r.m.s. radial velocity fluctuations in the shear layer. In all the jets, the shear-layer transition is dominated by vortex rolling-ups and pairings, which generate strong additional acoustic components, but also amplify the downstream-dominant low-frequency noise component when the exit boundary layer is thick. The introduction of inlet noise however results in weaker pairings, thus spectacularly reducing their contributions to the sound field. This high sensitivity to the initial conditions is in good agreement with experimental observations.

Key words: jet noise, shear layer turbulence, turbulence simulation

\section{Introduction}

Since many experimental works conducted during the 1970s including Maestrello \& McDaid (1971), Grosche (1974) and Hill, Jenkins \& Gilbert (1976), it has been well known that the flow development and the acoustic characteristics of axisymmetric jets strongly depend on the properties of the nozzle-exit boundary layer. In jet studies, parameters such as the momentum thickness of the velocity profile, the levels of velocity fluctuations, as well as the laminar or turbulent state of the boundary layer, have thus to be considered, because their variations may noticeably modify turbulent mixing and noise generation features. Unfortunately, they have been neither controlled nor documented in most experiments, except in a few papers such as Hussain \& Zedan 
(1978a,b), Husain \& Hussain (1979), Bridges \& Hussain (1987), Zaman (1985a,b) or more recently in Morris \& Zaman (2009). In round jets, Zaman (1985a,b) measured for instance an initial shear-layer momentum thickness of $\delta_{\theta}=0.008 r_{0}$, where $r_{0}$ is the jet radius, and negligible turbulent intensities at a diameter-based Reynolds number $R e_{D}=7 \times 10^{4}$, but $\delta_{\theta}=0.004 r_{0}$ and root-mean-square (r.m.s.) axial velocity fluctuations close to $10 \%$ of the jet velocity at $R e_{D}=2.5 \times 10^{5}$. In the same way, Bridges \& Hussain (1987) reported in jets at $R e_{D}=1.4 \times 10^{5}$ and $3.2 \times 10^{5}$ nozzleexit momentum thicknesses $\delta_{\theta}=0.005 r_{0}$ and $0.003 r_{0}$ and peak r.m.s. axial velocity fluctuations of 0.5 and $1.9 \%$, respectively.

One difficulty in experiments studying the effects of initial conditions is that the inflow parameters cannot usually be changed independently, except for some very careful investigations such as those by Hussain \& Zedan $(1978 a, b)$. To distinguish the influence of jet inflow parameters, it then appears natural to turn to numerical simulations. Works have been carried out to this end by Stanley \& Sarkar (2000), Bogey \& Bailly (2005), Bogey, Barré \& Bailly (2008) and Kim \& Choi (2009). However, some questions remain because the computing limitations have led to differences between experimental and numerical inflow conditions, as specifically highlighted in reviews by Colonius \& Lele (2004) and Bodony \& Lele (2008). The initial shear-layer momentum thicknesses specified in simulations, e.g. $\delta_{\theta}=0.016 r_{0}$ in Bogey et al. (2008) and $\delta_{\theta}$ between $0.011 r_{0}$ and $0.04 r_{0}$ in Kim \& Choi (2009), have in particular often been higher than corresponding experimental values. The discrepancies observed in many jet computations with respect to the measurements, such as too short potential cores and excessive sound pressure levels, have thus been believed to result from the use of artificially thickened shear layers.

According to experimental results, the influence of the initial shear-layer thickness on subsonic jet noise should however depend on the state of the turbulence at the nozzle exit. The variations of far-field noise features with the exit boundary-layer thickness can be expected to be stronger in initially laminar jets than in initially turbulent jets, namely tripped jets and jets at sufficiently high Reynolds numbers. Jets exhibit indeed laminar initial state for Reynolds numbers lower than a threshold value, which is around $R e_{D}=10^{5}$ from the analysis by Crighton (1981) based on a set of experimental data. In the untripped jets of Zaman $(1985 a, b)$ for example, the flows were initially laminar for $R e_{D} \leqslant 2.5 \times 10^{5}$. In such flows, rolling-ups and pairings of coherent vortical structures dominate the turbulent development in the first few diameters downstream of the nozzle. Because vortex pairings are efficient noise generators, initially laminar jets thus emit additional noise components with respect to initially turbulent jets as shown experimentally by Maestrello \& McDaid (1971), Grosche (1974), Zaman (1985a,b) and Bridges \& Hussain (1987) and numerically by Bogey et al. (2008), which may be predominant, especially for wide radiation angles. The sound field of low amplitude observed at such angles appears indeed to be generated by the turbulent scales developing randomly in the jets as suggested by Bogey \& Bailly $(2006 a, 2007)$ and Tam et al. (2008).

Following the discussion just above, the turbulent development and noise of laminar jets will naturally be affected by the variations of the exit boundary-layer thickness, or by the introduction of initial disturbances as in the tripped jets of Lepicovsky \& Brown (1989) and Russ \& Strykowski (1993) for instance, through direct modifications of pairing frequencies and strengths. In this study, the influence of the nozzle-exit boundary-layer conditions in initially laminar round jets is therefore investigated by performing large-eddy simulations (LES) combining low-dissipation and low-dispersion schemes and relaxation filtering for dissipating subgrid-scale 
energy. The acoustic far field radiated by the jets is calculated from the LES near field by solving the linear acoustic equations. The jets are isothermal, and at a Mach number of 0.9 and a Reynolds number of $10^{5}$. To get closer to the experiments, a part of a pipe nozzle is included in the computational domain, and laminar boundary-layer profiles of thicknesses between $\delta=0.025 r_{0}$ and $\delta=0.2 r_{0}$ are imposed at the nozzle inlet. In first simulations, no disturbance is added in the pipe nozzle to examine the influence of the nozzle-exit momentum thickness $\delta_{\theta}$ in initially fully laminar jets, down to typical experimental values. In two additional simulations, random disturbances of low amplitude are added in the jet nozzle to investigate the effects of small variations of the exit turbulence, especially on the early stage of the shear-layer transition and on the radiated noise. Thus, we will be able to exhibit the way in which changing $\delta_{\theta}$ or the turbulent conditions at the nozzle exit modifies noise components in initially laminar subsonic jets.

This paper is organized as follows. In $\S 2$, the parameters of the jet LES and the extrapolation of the LES near field to the far field, including numerical algorithm, computational grids and times, are documented. The initial conditions of the jets, as well as the flow conditions at the pipe exit, are also presented. The near-field results provided by LES are described in $\S 3$. Vorticity and pressure snapshots are represented, and comparisons with measurements for initially laminar jets are shown. The shear-layer transitions and the overall flow developments of the different jets are then studied in detail. The far-acoustic fields determined using an extrapolation method are examined and discussed in $\S 4$. Concluding remarks are finally provided in $\S 5$. The coefficients used for a non-centred filter are given in Appendix A, and results obtained from additional simulations are shown in Appendices B and $\mathrm{C}$ to illustrate the very small influence of the numerical parameters on the flow and the noise fields reported in this work.

\section{Study parameters}

\subsection{Jet definition}

Circular isothermal jets at Mach number $M=u_{j} / c_{a}=0.9$ and Reynolds number $R e_{D}=u_{j} D / v=10^{5}$, originating from a pipe nozzle of radius $r_{0}$ and length $1.1 r_{0}$, are computed by LES ( $u_{j}$ is the jet inflow velocity, $c_{a}$ is the speed of sound in the ambient medium, $D=2 r_{0}$ is the nozzle diameter and $v$ is the kinematic molecular viscosity). The ambient temperature and pressure are $293 \mathrm{~K}$ and $10^{5} \mathrm{~Pa}$, respectively. At the exit section of the nozzle at $z=0$, the width of the nozzle lip is $0.053 r_{0}$. At the pipe inlet at $z=-1.1 r_{0}$, laminar Blasius boundary layers of thickness $\delta$ are imposed. The profiles of the axial velocity $u_{z}$ are more precisely given by a polynomial approximation of the Blasius profile in the following way:

$$
\begin{aligned}
& u_{z}(r)=u_{j} \frac{r_{0}-r}{\delta}\left[2-2\left(\frac{\left(r_{0}-r\right)}{\delta}\right)^{2}+\left(\frac{r_{0}-r}{\delta}\right)^{3}\right], \quad \text { if } \quad r \geqslant r_{0}-\delta, \\
& u_{z}(r)=u_{j}, \quad \text { if } \quad r<r_{0}-\delta,
\end{aligned}
$$

where $r$ is the distance from the jet centreline. In addition, the radial and azimuthal velocities are initially set to zero, pressure is kept constant to the ambient pressure and the temperature is determined by a Crocco-Busemann relation.

Four jets characterized by inlet boundary-layer thicknesses $\delta=0.025 r_{0}, 0.05 r_{0}, 0.1 r_{0}$ and $0.2 r_{0}$, referred to as JetD0025, JetD005, JetD01 and JetD02, respectively, are simulated as reported in table 1 . To better match experiments, in which jets even at 


\begin{tabular}{llcc}
\hline Reference & $\delta / r_{0}$ & Inlet random noise & Line style \\
JetD02 & 0.2 & - & - \\
JetD01 & 0.1 & - & -- \\
JetD005 & 0.05 & - & --- \\
JetD0025 & 0.025 & $250 \mathrm{~Pa}$ & - \\
JetD005p250 & 0.05 & $2000 \mathrm{~Pa}$ & ---
\end{tabular}

TABLE 1. Thickness of the inlet Blasius boundary layer $\delta$, maximum amplitude of possible random pressure disturbances in the pipe and line styles used in the plots.

moderate Reynolds number may rarely be fully initially laminar, two additional jets with an exit boundary-layer thickness $\delta=0.05 r_{0}$ are calculated. In these two cases JetD005p250 and JetD005p2000, unlike the four previous jets in which no forcing is applied in the nozzle, random pressure disturbances are introduced in the pipe within the boundary layer between $z=-0.4 r_{0}$ and $z=-0.2 r_{0}$. They are of maximum amplitudes of 250 and $2000 \mathrm{~Pa}$, respectively, which is low enough so that the impact on the acoustic fields is negligible, as shown in Appendix C. Finally, in order to seed the turbulent transition at the beginning of the simulations, random pressure fluctuations of maximum amplitude $200 \mathrm{~Pa}$ are added in the shear layer between $z=0.25 r_{0}$ and $z=4 r_{0}$ up to non-dimensional time $t=18.75 \mathrm{D} / u_{j}$ in the six jet LES.

\subsection{LES procedure and parameters}

The LES is performed using a solver of the three-dimensional filtered compressible Navier-Stokes equations developed in cylindrical coordinates $(r, \theta, z)$ using lowdissipation and low-dispersion finite-difference schemes. The axis singularity is taken into account by the method proposed by Mohseni \& Colonius (2000). Fourth-order 11-point centred finite differences are used for spatial discretization, and a second-order six-stage low-storage Runge-Kutta algorithm is implemented for time integration; see Bogey \& Bailly (2004). To circumvent the severe time-step restriction induced by the cylindrical coordinates, the derivatives in the azimuthal direction around the axis are calculated using every $n$th grid point, from $n=2$ up to $n=32$ at the closest points to the axis. To remove grid-to-grid oscillations, a sixth-order 11-point centred filtering designed by Bogey, de Cacqueray \& Bailly (2009b) to damp only the shortest waves discretized is applied every time step to the flow variables. The discretization at the boundaries is also taken into account by noncentred finite differences and filters with properties optimized in the Fourier space, given by Berland et al. (2007b) (see Appendix A for the coefficients of a modified non-centred filter). The filtering is also employed to dissipate subgrid-scale energy without significantly affecting the scales resolved accurately. This LES approach was developed not to artificially decrease the effective flow Reynolds number, which might be the case using eddy-viscosity subgrid models. More details on this approach based on relaxation filtering, which can be referred to as LES-RF, are available in Bogey \& Bailly $(2006 b, c, 2009)$. Finally, in order to compute the jet noise directly, the radiation boundary conditions developed by Tam \& Dong (1996) and Bogey \& Bailly (2002) are specified, with the addition of a sponge zone at the outflow. These non-reflective conditions are also applied at the pipe inlet. In this way, acoustic resonance inside the nozzle is unlikely, specially when pressure disturbances are added in the pipe as shown by the results presented in Appendix C for an additional simulation without flow. 


\begin{tabular}{lcrccccc}
\hline Reference & $n_{r} \times n_{\theta} \times n_{z}$ & $n_{r}^{\text {pipe }}$ & $n_{z}^{\text {pipe }}$ & $\Delta r\left(r=r_{0}\right)$ & $\Delta z(z=0)$ & $n_{t}$ & $T u_{j} / D$ \\
JetD02 & $173 \times 256 \times 505$ & 70 & 20 & $0.028 r_{0}$ & $0.056 r_{0}$ & 48800 & 275 \\
JetD01 & $215 \times 256 \times 543$ & 94 & 27 & $0.014 r_{0}$ & $0.028 r_{0}$ & 46800 & 275 \\
JetD005 & $249 \times 256 \times 595$ & 124 & 35 & $0.007 r_{0}$ & $0.014 r_{0}$ & 86000 & 250 \\
JetD0025 & $287 \times 256 \times 651$ & 154 & 44 & $0.0035 r_{0}$ & $0.007 r_{0}$ & 127800 & 187.5
\end{tabular}

TABLE 2. Numbers of grid points $\left(n_{r}, n_{\theta}, n_{z}\right)$, points within the pipe $n_{r}^{\text {pipe }}$ and $n_{z}^{\text {pipe }}$, mesh spacings at the pipe lip, time steps $n_{t}$, and whole simulation time $T$. The parameters for JetD005p250 and JetD005p2000 are the same as those for JetD005.

(a)

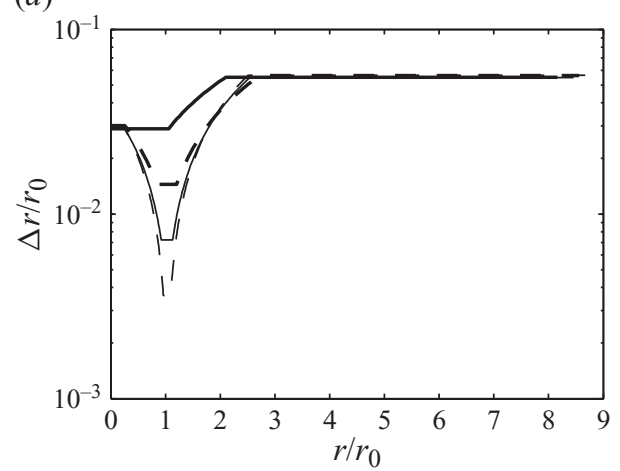

(b)

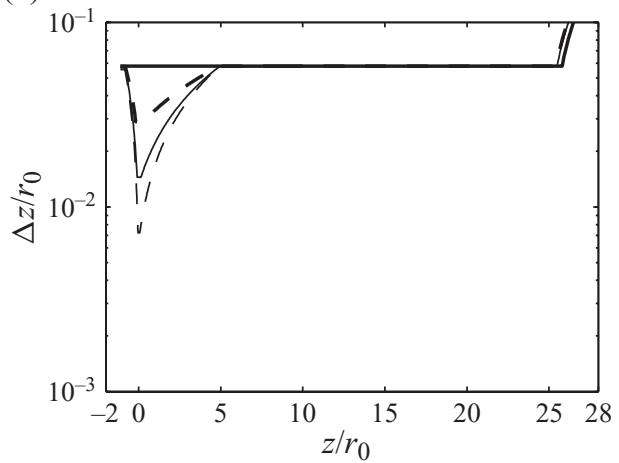

FIGURE 1. Mesh spacings in the $(a)$ radial and $(b)$ axial directions, $\Delta r$ and $\Delta z$, in the LES, for — JetD02, - - - JetD01, — JetD005, - - JetD0025, — JetD005p250 and --- JetD005p2000.

The main parameters of the LES grids, as well as the numbers of time steps and the non-dimensional simulation times $T u_{j} / D$ of the different simulations, are provided in table 2 . The grids extend radially up to $r \simeq 8.6 r_{0}$, and the sponge zones are built from the axial position $z=25.5 r_{0}$. They contain from 22 to 48 million points, and are characterized by the radial and axial mesh spacings $\Delta r$ and $\Delta z$ shown in figure 1 . The mesh grids are adjusted to the varying exit boundary-layer thicknesses by specifying minimum mesh spacings at the nozzle lip $\Delta r=\delta / 7$ and $\Delta z=2 \delta / 7$, yielding $\Delta r=0.028 r_{0}$ in JetD02 down to $\Delta r=0.0035 r_{0}$ in JetD0025. They are then stretched at rates lower than $4 \%$ to reach maximum values $\Delta r=\Delta z=0.056 r_{0}$ in the physical computational domains. This mesh size is chosen so that the time frequency $f$ of waves discretized by four grid points corresponds to Strouhal number $S t=f D / u_{j}=10$. Grid stretching enables avoidance of excessive numbers of grid points in the pipe. There are for example only 154 points within the pipe diameter in JetD0025, which is still affordable compared to the 560 points that would be required using a uniform grid. At this point, note that the influence of the grid resolution and the sponge zone has been investigated in Appendices B and C, by performing two additional simulations at the same inflow conditions as in JetD02: one using the mesh defined for the JetD01 jet which is twice as fine in the axial and radial directions at the nozzle lip, yielding for example $\Delta r=\delta / 14$ instead of $\Delta r=\delta / 7$ at $r=r_{0}$, and another with a sponge zone beginning at $z=20.5 r_{0}$ instead of $z=25.5 r_{0}$. Neither the flow nor the sound field have been found to be significantly modified.

For the study of jet turbulence features and the far-field acoustic extrapolation, density, velocity components and pressure are recorded from time $t=37.5 \mathrm{D} / u_{j}$ at 


\begin{tabular}{lcccccc}
\hline & $\delta_{\theta} / r_{0}$ & $\delta_{\theta} / r_{0}$ & $\left\langle u_{z}^{\prime 2}\right\rangle^{1 / 2} / u_{j}$ & $\max \left(\left\langle u_{z}^{\prime 2}\right\rangle^{1 / 2}\right) / u_{j}$ & $L_{11}^{(\theta)}$ & $\max \left(L_{11}^{(\theta)}\right)$ \\
Reference & at $z=0$ & at $z=0.4 r_{0}$ & at $z=r=0$ & at $z=0$ near $r=r_{0}$ & at $z=0.1 r_{0}$ & at $r=r_{0}$ \\
JetD02 & 0.0232 & 0.0246 & 0.0026 & 0.0017 & $0.59 \pi$ & $0.79 \pi$ \\
JetD01 & 0.0116 & 0.0128 & 0.0054 & 0.0023 & $0.61 \pi$ & $0.65 \pi$ \\
JetD005 & 0.0056 & 0.0072 & 0.0059 & 0.0031 & $0.53 \pi$ & $0.59 \pi$ \\
JetD0025 & 0.0025 & 0.0048 & 0.0048 & 0.0036 & $0.40 \pi$ & $0.44 \pi$ \\
JetD005p250 & 0.0056 & 0.0072 & 0.0041 & 0.0032 & $0.39 \pi$ & $0.41 \pi$ \\
JetD005p2000 & 0.0056 & $0.0060\left(^{*}\right)$ & 0.0045 & 0.0192 & $0.06 \pi$ & $0.06 \pi$
\end{tabular}

TABLE 3. Shear-layer momentum thickness $\delta_{\theta}$ at $z=0$ and at $z=0.4 r_{0}$, except for $\left(^{*}\right)$ at $z=0.1 r_{0}$, centreline and maximum near-wall r.m.s. values of the axial fluctuating velocity $u_{z}^{\prime}$ at the pipe exit at $z=0$, and integral length scales in the azimuthal direction $L_{11}^{(\theta)}$ calculated from $u_{z}^{\prime}$ along the lip line at $r=r_{0}$, in radians.

(a)

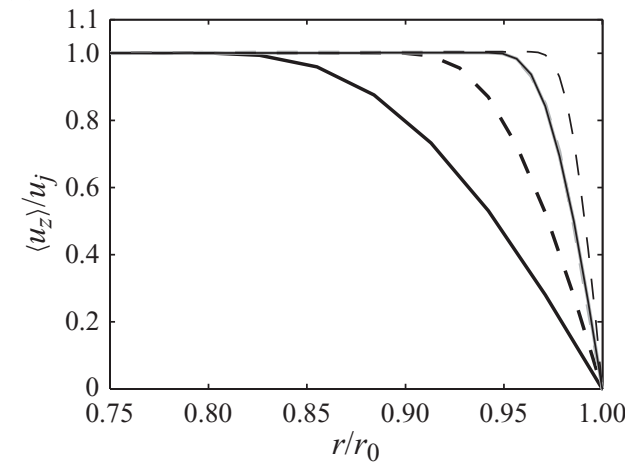

(b)

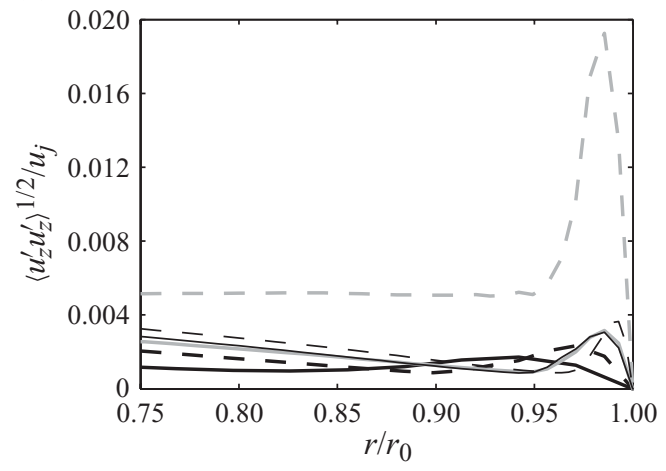

FIGURE 2. Profiles at $z=0$ of $(a)$ the mean axial velocity $\left\langle u_{z}\right\rangle$ and $(b)$ the r.m.s. values of fluctuating axial velocity $u_{z}^{\prime}$, for $\longrightarrow$ JetD02, - - JetD01, JetD005, - - JetD0025, — JetD005p250 and - - JetD005p2000.

every point along the centreline at $r=0$, and on cylindrical surfaces located at $r=r_{0}$ and at $r=5.25 r_{0}$, at a frequency allowing computation of spectra up to Strouhal number 10 . The velocity spectra are evaluated from overlapping samples of duration $10 D / u_{j}$. The flow statistics are also determined from $t=87.5 D / u_{j}$, and results are averaged in the azimuthal direction.

\subsection{Nozzle-exit conditions}

The flow conditions obtained at the nozzle-pipe exit in the jet LES are presented. They are first illustrated in figure 2 with the mean and r.m.s. turbulent profiles calculated for the axial velocity at $z=0$. The profiles of the mean velocity $\left\langle u_{z}\right\rangle$ agree with the Blasius profiles specified at the pipe inlet, as expected because the boundary layers do not develop in the short pipe, while the fluctuation intensities are of amplitude lower than $2 \%$. The momentum thicknesses $\delta_{\theta}$ determined from $\left\langle u_{z}\right\rangle$ at $z=0$ at the exit plane are provided in table 3 . They range from $0.0025 r_{0}$ for JetD0025 to $0.0232 r_{0}$ for JetD02. As for the r.m.s. levels of fluctuating velocity $u_{z}^{\prime}$, maximum values near the wall are around $0.003 u_{j}$ for all jets except for JetD005p2000, in which the peak is about $0.02 u_{j}$. Following Zaman $(1985 a, b)$, the initial boundary layers of the jets are therefore fully laminar for the four jets without inlet noise and for JetD005p250, and nominally laminar for JetD005p2000. In all jets, the velocity fluctuations on the centreline are 
(a)

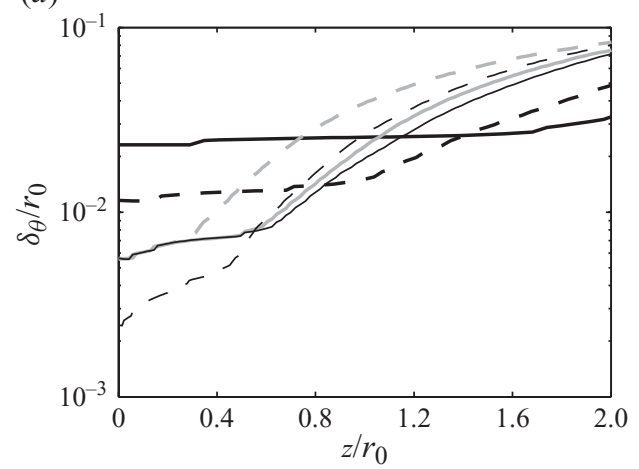

(b)

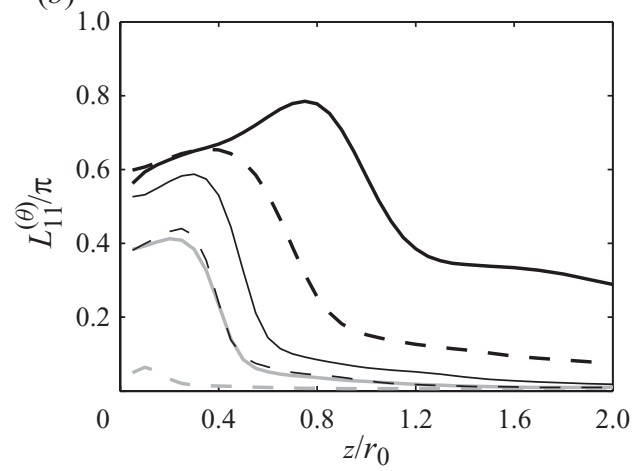

FIGURE 3. Axial variations in the vicinity of the pipe lip, of $(a)$ the shear-layer momentum thickness $\delta_{\theta}$ and $(b)$ the integral length scale in the azimuthal direction $L_{11}^{(\theta)}$ calculated from the fluctuating axial velocity at $r=r_{0}$, for $\longrightarrow$ JetD02, $---J e t D 01,-J e t D 005$, - - - JetD0025, JetD005p250 and - - JetD005p2000.

also observed to be of low amplitude, between 0.2 and $0.6 \%$ of the jet velocity. To finally compare with some experiments of the literature dealing with jet noise, it can be noted that Zaman (1985a,b) and Bridges \& Hussain (1987) measured in jets at Reynolds numbers $R e_{D}=10^{5}$ and $R e_{D}=1.4 \times 10^{5}$, respectively, initial momentum thicknesses of $\delta_{\theta}=0.006 r_{0}$ and $\delta_{\theta}=0.005 r_{0}$, and turbulent intensities around $0.5 \%$. These conditions are quite similar to those in JetD005 and JetD005p250.

To further characterize the flow just downstream of the nozzle lip, the variations of the shear-layer momentum thickness $\delta_{\theta}$ and of the integral length scale $L_{11}^{(\theta)}$ of fluctuating axial velocity $u_{z}^{\prime}$ in the azimuthal direction are presented in figure 3 for $0 \leqslant z \leqslant 2 r_{0}$. The momentum thickness is estimated from the mean axial velocity $\left\langle u_{z}\right\rangle$ as

$$
\delta_{\theta}=\int_{0}^{r_{0.04}} \frac{\left\langle u_{z}\right\rangle}{u_{c}}\left(1-\frac{\left\langle u_{z}\right\rangle}{u_{c}}\right) \mathrm{d} r
$$

where $\langle$.$\rangle denotes statistical averaging, u_{c}$ is the centreline mean axial velocity, and $r_{0.04}$ is determined so that $\left\langle u_{z}\right\rangle\left(r=r_{0.04}\right)=0.04 u_{c}$. The azimuthal integral length scale is evaluated as

$$
L_{11}^{(\theta)}=\frac{1}{r_{0}} \int_{0}^{\pi} \mathscr{R}_{11}^{(\theta)} \mathrm{d}\left(r_{0} \delta \theta\right)
$$

from the cross-correlation function $\mathscr{R}_{11}^{(\theta)}$ of velocity $u_{z}^{\prime}$ defined at point $(r, \theta, z)$ by

$$
\mathscr{R}_{11}^{(\theta)}(\delta \theta)=\frac{\left\langle u_{z}^{\prime}(r, \theta, z) u_{z}^{\prime}(r, \theta+\delta \theta, z)\right\rangle}{\left\langle u_{z}^{\prime 2}(r, \theta, z)\right\rangle^{1 / 2}\left\langle u_{z}^{\prime 2}(r, \theta+\delta \theta, z)\right\rangle^{1 / 2}},
$$

where $\delta \theta$ is the azimuthal separation. The length scale, calculated at $r=r_{0}$, is normalized by $r_{0}$ to be in radians. For the axisymmetric mode, $L_{11}^{(\theta)}=\pi$ would be obtained for instance.

The variations of $\delta_{\theta}$ in figure 3 display two distinct stages typical of the development of initially laminar shear layers, as previously observed experimentally by Hussain \& Zedan $(1978 a, b)$ and Husain \& Hussain (1979). The momentum thickness first grows slowly during the flow adjustment following the nozzle lip, from a boundary-layer 
velocity profile to a shear-layer velocity profile, then increases rapidly when vortical structures appear, as will be evidenced later by the snapshots of figure 6 . The second stage of shear-layer development takes place earlier when the nozzle exit $\delta_{\theta}$ is smaller, which is in agreement with the linear instability theory of Michalke (1984) predicting that instability waves grow at higher rates for sharper velocity gradients, and when inlet noise is introduced. Moreover, during the first stage, the laminar shear layer spreads in a negligible manner in JetD02, but more significantly as the initial momentum thickness becomes smaller, which is certainly due to molecular viscosity. Given the appreciable variations of $\delta_{\theta}$ during the early transition, the values of momentum thickness which will be used later as the initial shear-layer thickness are therefore taken at $z=0.1 r_{0}$ for JetD005p2000, and at $z=0.4 r_{0}$ for all other jets. They are provided in table 3 .

The azimuthal integral length scales $L_{11}^{(\theta)}$ at $r=r_{0}$ exhibit similar axial variations in figure 3, with a slight increase just downstream of the exit plane, rapidly followed by a collapse down to much smaller values. Peak and initial values at $z=0.1 r_{0}$ are reported in table 3 . In the vicinity of the nozzle exit, length scales up to $0.59 \pi$ are noticed, which indicates that azimuthal correlation is high in the present laminar shear layers. The addition of random pressure disturbances in the pipe however appears to lower the initial azimuthal correlations: from $L_{11}^{(\theta)}=0.53 \pi$ for JetD005, one gets $L_{11}^{(\theta)}=0.39 \pi$ for JetD005p 250 , and only $L_{11}^{(\theta)}=0.06 \pi$ for JetD005p2000. Now, it can be worth pointing out that the nozzle-exit conditions in JetD005 and JetD005p250 are very close: mean velocity profiles and turbulent intensities are the same, but the azimuthal velocity correlations differ.

\subsection{Far-field extrapolation}

For characterization of the noise generated by the jets, and comparison with experimental data, the near field obtained directly by LES is propagated to 60 radii from the nozzle exit, where far-field conditions are expected to apply to the acoustic field according to the experimental results of Ahuja, Tester \& Tanna (1987). Sound propagation is carried out by solving the linear acoustic equations written in cylindrical coordinates, for the fluctuating velocity components and pressure as done previously by Berland, Bogey \& Bailly (2007a) and Bogey et al. (2009a). The numerical schemes and boundary conditions used are the same as those implemented in the LES. Non-centred finite differences and filters are applied at the inner-side boundary of the extrapolation grid where the LES data are introduced.

In practice, far-field extrapolation is performed from LES velocities and pressure at $r=5.25 r_{0}$, as mentioned in $\S 2.2$. These data are interpolated on a cylindrical surface discretized by a uniform mesh spacing $\Delta z=0.056 r_{0}$ in the axial direction, thus containing 450 points from $z=-0.6 r_{0}$ to $z=25 r_{0}$. They are imposed at the bottom boundary of a cylindrical grid of $n_{r} \times n_{\theta} \times n_{z}=751 \times 256 \times 881$ points, extending up to $z=54 r_{0}$ and $r=61.5 r_{0}$, on which the linear acoustic equations are solved to propagate noise. The mesh spacings of the extrapolation grid are shown in figure 4 . The maximum mesh size is $\Delta r=\Delta z=0.075 r_{0}$, yielding Strouhal number $S t=7.4$ for the sound waves discretized by four grid points. After a propagation time $t=30 \mathrm{D} / \mathrm{u}_{j}$, pressure is recorded around the jets at points located at $60 r_{0}$ from $z=r=0$, during time periods of $225 \mathrm{D} / u_{j}$ for JetD02 and JetD01, 200D/ $u_{j}$ for JetD005, JetD005p250 and JetD005p2000, and $135 D / u_{j}$ for JetD0025. Spectra are evaluated from the pressure signals using overlapping samples of duration $30 D / u_{j}$, and they are averaged in the azimuthal direction. 


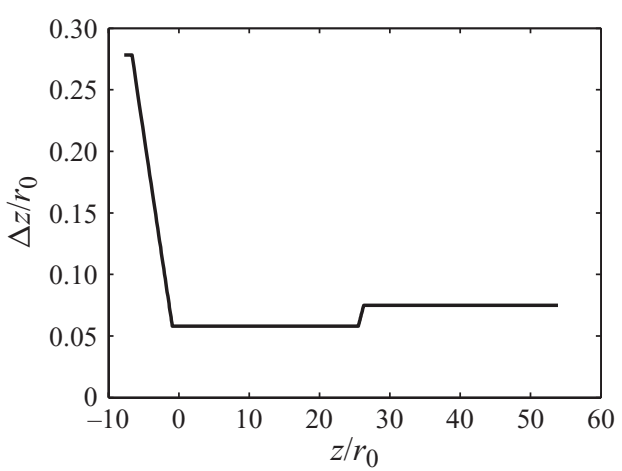

FIGURE 4. Mesh spacing in the axial direction $\Delta z$ in the acoustical simulations. The mesh spacing in the radial direction is $\Delta r=0.075 r_{0}$.

\section{Near-field results}

This section presents the effects of the initial conditions on the jet near field, by showing vorticity and pressure snapshots, and the main characteristics of the mean and turbulent developments of the shear layers and jet flows. Results are also compared to measurements obtained for initially laminar jets. Comparisons with experimental data available for jets at Mach number 0.9, such as those by Lau, Morris \& Fisher (1979), Arakeri et al. (2003) and Fleury et al. (2008), are not shown, because these jets are at Reynolds numbers higher than $5 \times 10^{5}$. Therefore, albeit possibly initially laminar, they are all likely to contain significant inflow turbulence. This is the case for instance in the jet of Arakeri et al. (2003), in which the peak r.m.s. values of $u_{z}^{\prime}$ at the exit section are around $10 \% u_{j}$, which is much higher than in the jets considered in this study.

\subsection{Vorticity and pressure snapshots}

Snapshots of vorticity norm obtained for the present jets are displayed in figure 5, as well as in supplementary movie 1 (available at journals.cambridge.org/flm) for the jets without inlet noise. In the initially laminar shear layers, the turbulent transition seems to be first dominated by coherent structures; then three-dimensional fine-scale turbulence is generated. Farther downstream, the mixing layers merge and turbulent jets are observed. From these overall jet pictures, it is difficult to clearly see the differences between the jet developments. Some effects of the nozzle-exit boundarylayer thickness are however visible, especially in supplementary movie 1, by comparing for instance the vorticity fields in JetD02 and JetD0025. For smaller initial $\delta_{\theta}$, vortical structures appear to form more rapidly in the shear layers. The mixing layers, once turbulent, also develop more slowly, leading to an increase of the potential core length, around $10 r_{0}$ in JetD02 versus $18 r_{0}$ in JetD0025.

To focus on the early jet flow development, vorticity snapshots obtained in the shear layers close to the exit plane are shown in figure 6 and supplementary movies 2 and 3. In the four jets without inlet noise, JetD02, JetD01, JetD005 and JetD0025 (see supplementary movie 2 in particular), the mechanisms taking place downstream of the nozzle lip are the same. The turbulent transition in the initially laminar mixing layers first consists of the processes of vortex rolling-up and pairing, whatever the exit boundary-layer thickness may be, then three-dimensional turbulence appears after the first vortex pairing. The variations of the initial momentum thickness affect the size of the coherent structures generated by the shear-layer rolling-up. As $\delta_{\theta}$ decreases, these 
(a)

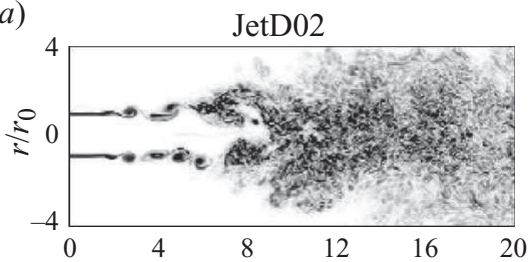

(c)
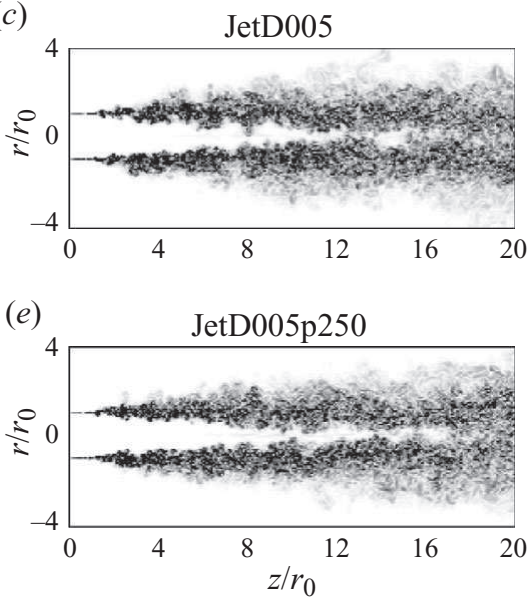

(b)

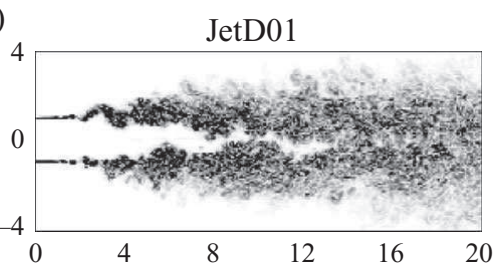

(d)

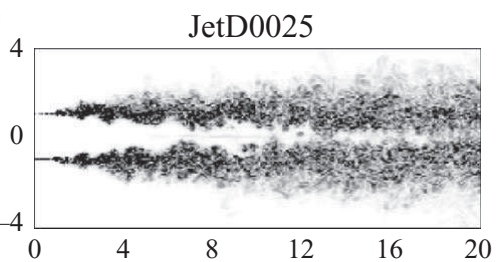

$(f)$

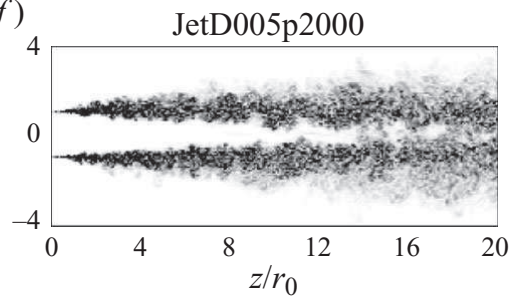

Figure 5. $(a-f)$ Snapshots in the $(z, r)$ plane of vorticity norm. The grey scale ranges from 0 up to the level of $6.5 u_{j} / r_{0}$, from white to black.
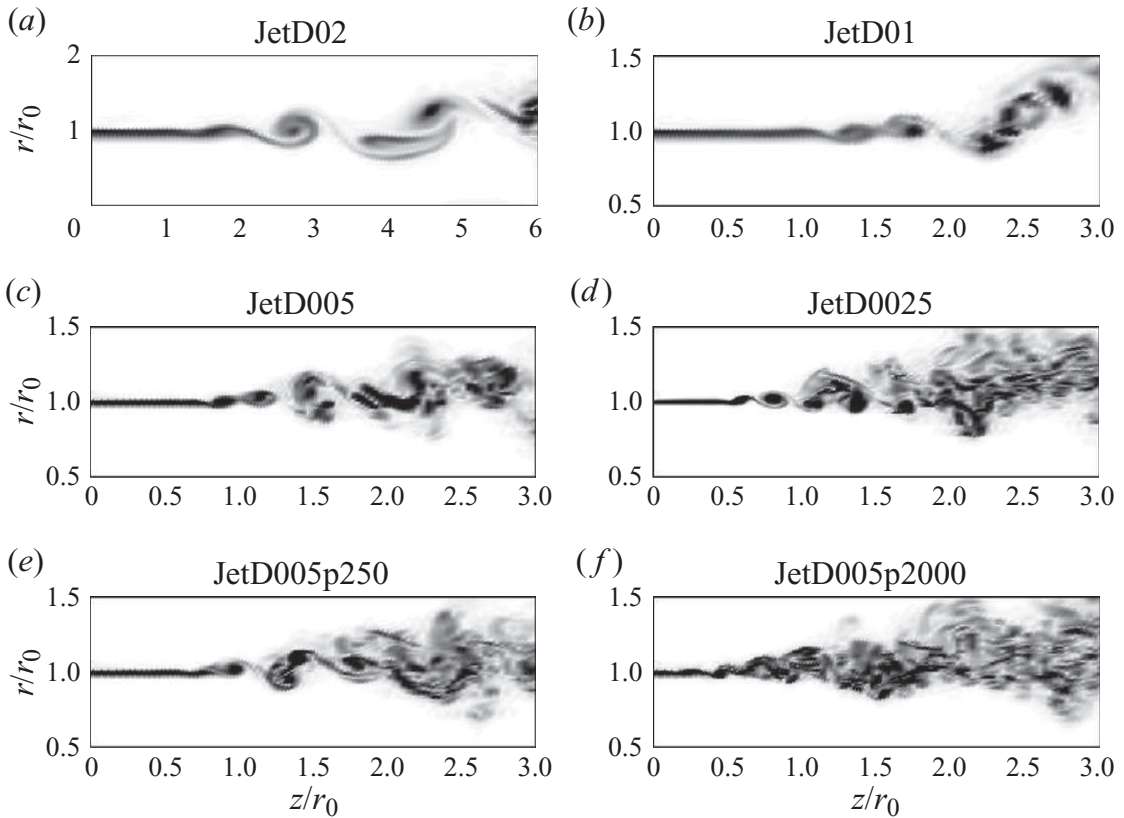

FiguRE 6. $(a-f)$ Snapshots in the $(z, r)$ plane of vorticity norm downstream of the pipe lip. The grey scale ranges from 0 up to the level of $10 u_{j} / r_{0}$ for JetD02, but up to $20 u_{j} / r_{0}$ for the other jets, from white to black. 

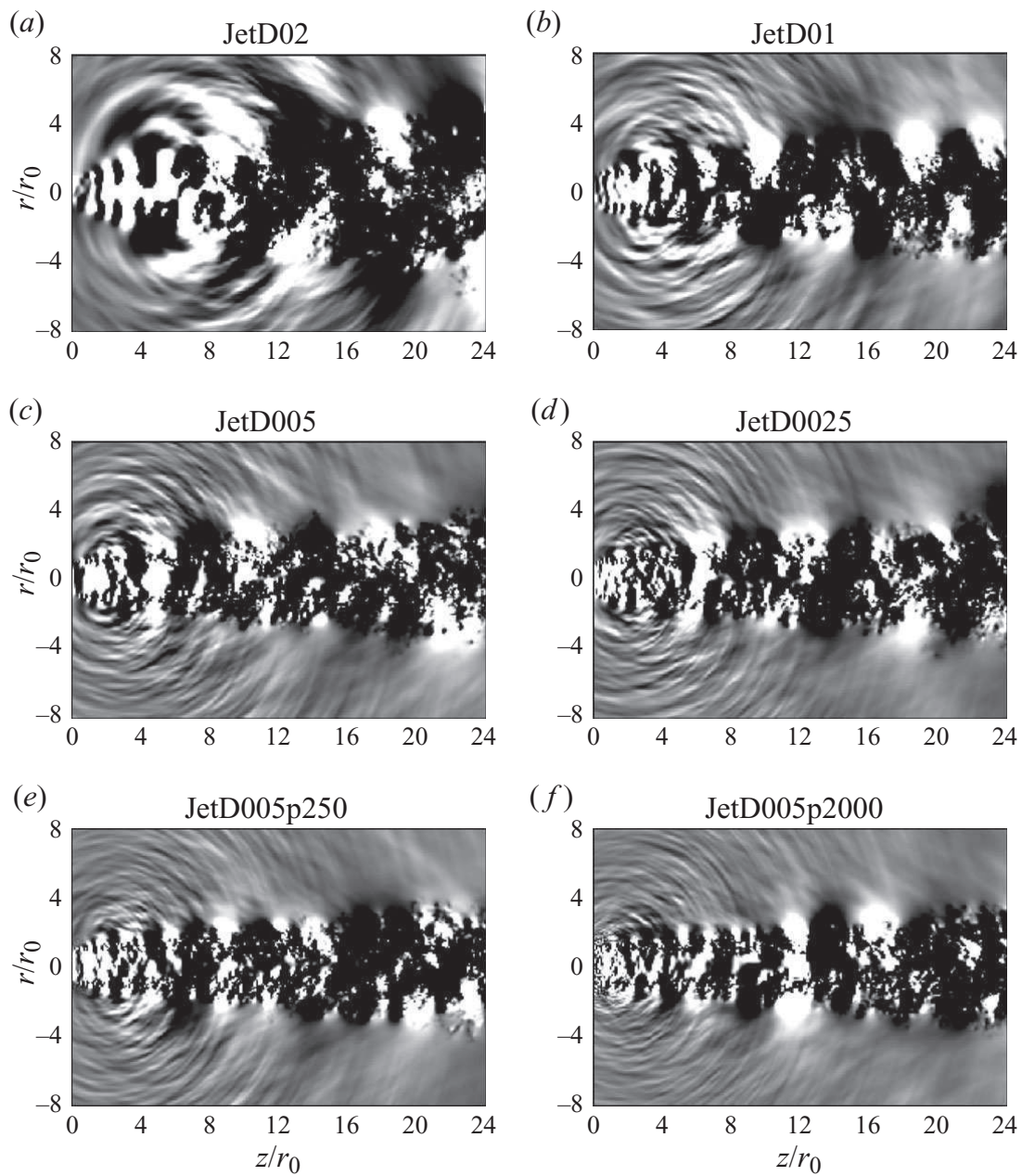

FIGURE 7. $(a-f)$ Snapshots in the $(z, r)$ plane of the fluctuating pressure obtained by LES. The grey scale ranges for levels from -250 to $250 \mathrm{~Pa}$, from black to white.

structures are significantly smaller, as expected. The rolling-up also occurs farther upstream. Finally, the vorticity fields obtained for JetD005p250 and JetD005p2000, in figure 6 and supplementary movie 3, are fairly similar to that for JetD005 with same initial $\delta_{\theta}$. In the two former jets, however, the mixing layers seem to develop somewhat earlier while exhibiting less organized vortical structures. This suggests that the rolling-up/pairing process may be hindered by the addition of low random noise inside the pipe, which will be demonstrated quantitatively in the next section.

As first illustrations of the noise generated by the jets, snapshots of the near pressure fields determined directly by LES are shown in figure 7 and supplementary movies 4 and 5. In JetD02, JetD01, JetD005 and JetD0025, strong acoustic waves are seen to propagate at wide angles, typically between 60 and $90^{\circ}$, relative to the jet direction. These waves visibly come from the transition region of the shear layers. Their apparent origins, which are closer to the jet exit as the initial shear-layer thickness decreases, even correspond roughly to the locations of the first vortex pairing in the mixing layers. Additionally, their associated wavelengths and levels are found both 


\begin{tabular}{llccc}
\hline Reference & $M$ & $R e_{D} \times 10^{-5}$ & $\delta_{\theta} / r_{0} \times 10^{3}$ & $u_{z}^{\prime} / u_{j}$ \\
Husain \& Hussain (1979) & 0.09 & 2.5 & 3.3 & 0.025 \\
Russ \& Strykowski (1993) & 0.02 & 0.1 & 23 & $<0.0025$ \\
Raman, Zaman \& Rice (1989) & 0.3 & 6 & 8 & 0.05
\end{tabular}

TABLE 4. Experiments on initially laminar jets: Mach and Reynolds numbers, initial shear-layer momentum thickness and maximum intensity of the velocity $u_{z}^{\prime}$ at the nozzle exit.

(a)

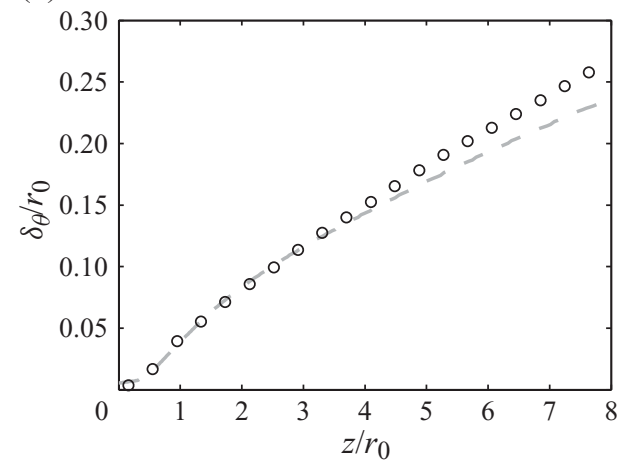

(b)

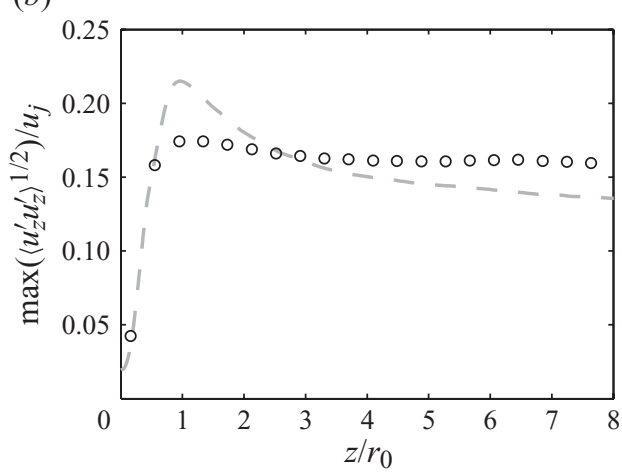

Figure 8. Variations of $(a)$ the shear-layer momentum thickness $\delta_{\theta}$ and $(b)$ the peak r.m.s. value of fluctuating velocity $u_{z}^{\prime}$ for $=--$ JetD005p2000. Measurements: $\bigcirc$, Husain \& Hussain (1979).

to be significantly reduced when smaller $\delta_{\theta}$ is specified at the nozzle exit. Concerning the near pressure fields obtained for JetD005p250 and JetD005p2000, they display features similar to those for JetD005. The acoustic waves radiated by these two jets are however of lower amplitude, as illustrated by supplementary movie 5.

\subsection{Comparisons with measurements}

Making comparisons between the simulation results and measurements can be tricky, because they might be meaningless if the jet inflow conditions are significantly different. Fortunately there are some experiments on initially laminar jets in which the boundary-layer thickness and turbulent intensities at the nozzle exit are described, and roughly correspond to the initial conditions of the present jets. This is the case in the works of Husain \& Hussain (1979), Russ \& Strykowski (1993) and Raman et al. (1989), whose main parameters, including Mach and Reynolds numbers, are provided in table 4 .

Husain \& Hussain (1979) considered an untripped axisymmetric mixing layer initially characterized by a momentum thickness $\delta_{\theta}=0.0033 r_{0}$ and by maximum axial turbulent intensities of $2.5 \%$. These values are rather close to the exit conditions reported in table 3 for JetD005p2000, which are $\delta_{\theta}=0.0056 r_{0}$ and peak r.m.s. velocities $u_{z}^{\prime}$ of $0.019 u_{j}$. The properties of the mixing layer developing in JetD005p2000 are therefore compared with the data of Husain \& Hussain (1979) in figure 8. The agreement is satisfactory given that the exit boundary-layer conditions in the simulation and the experiment are not exactly the same, and that the Mach number of the jet in Husain \& Hussain (1979) is only 0.09. The variations of the shear-layer momentum thickness are specially similar, whereas the profiles obtained for the axial 
(a)

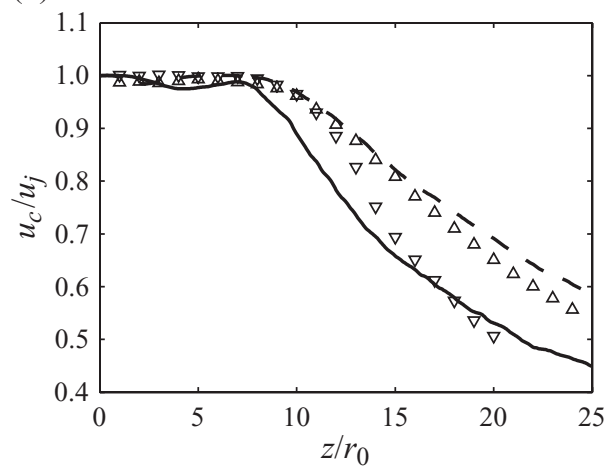

(b)

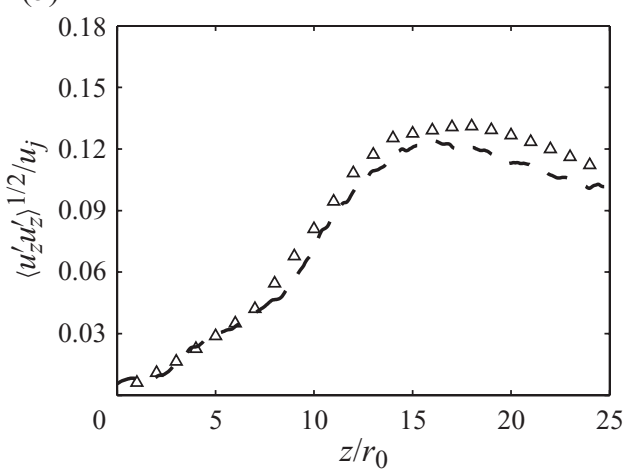

FIGURE 9. Variations of $(a)$ the mean axial velocity $u_{c}$ and $(b)$ the r.m.s. values of fluctuating velocity $u_{z}^{\prime}$ along the centreline, for — JetD02, - - - JetD01. Measurements: $\nabla$, Russ \& Strykowski (1993); $\triangle$, Raman et al. (1989).

turbulent intensities differ noticeably. The peak of turbulent intensities is indeed higher in the simulation, which may be partly due to lower initial turbulence levels.

Concerning the flow field of fully initially laminar round jets, experimental works were carried out by Russ \& Strykowski (1993) and Raman et al. (1989) for jets exhibiting laminar nozzle-exit boundary layers of thickness $\delta_{\theta}=0.023 r_{0}$ and $\delta_{\theta}=0.008 r_{0}$, respectively, as shown in table 4 . These conditions are nearly identical to the exit parameters in JetD02 and JetD01 collected in table 3, which are $\delta_{\theta}=0.0232 r_{0}$ and $\delta_{\theta}=0.0116 r_{0}$, respectively, for the boundary-layer thickness. The centreline variations of the mean axial velocity and of the r.m.s. values of velocity $u_{z}^{\prime}$ from JetD02 and JetD01 are consequently represented in figure 9 along with the results from the experiments, when available. The velocity decays from JetD02 and Russ \& Strykowski (1993) are first comparable, the potential core being however longer in the experiment maybe because of a lower Reynolds number of $10^{4}$. The mean and turbulent developments of the jets from JetD01 and Raman et al. (1989) also fairly agree. Thus, for similar exit boundary-layer conditions notwithstanding variations in terms of Mach and Reynolds numbers in particular, simulations and experiments appear to yield solutions that correspond relatively well.

\subsection{Shear-layer development}

The growth of the shear layer in the different simulated jets is first investigated by displaying in figure 10 the variations of the momentum thickness $\delta_{\theta}$ and of the spreading rate $\mathrm{d} \delta_{\theta} / \mathrm{d} z$ for $z \leqslant 8 r_{0}$. As the initial momentum thickness becomes smaller, according to the graphs of $\delta_{\theta}$, the shear layers develop earlier, but more slowly. This observation is supported quantitatively by the spreading rates, which reach peaks more rapidly, but display high values over shorter axial distances for thinner shear layers. This behaviour is moreover strengthened by the addition of inlet random noise inside the nozzle.

The intensities of turbulence in the shear layers are shown in figure 11 with the variations of the levels of fluctuating axial, radial and azimuthal velocities, and of the Reynolds shear stress along the lip line. In agreement with previous results, they grow more rapidly in thinner shear layers, and when random noise is introduced upstream. For all components except azimuthal velocity $u_{\theta}^{\prime}$, the curves obtained for JetD02, JetD01, JetD005 and JetD0025 exhibit dual-peak shapes, which is typical, 
(a)

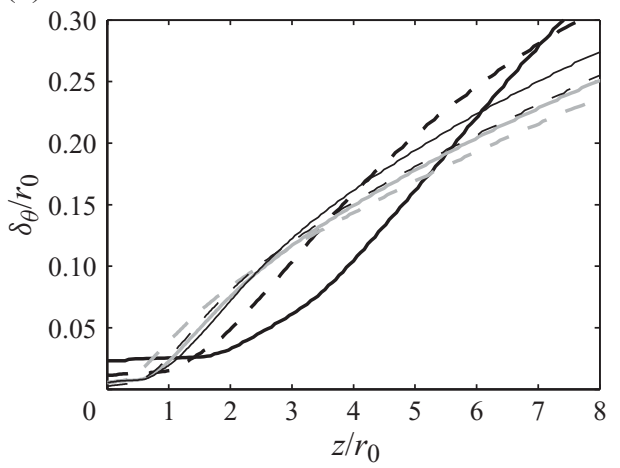

(b)

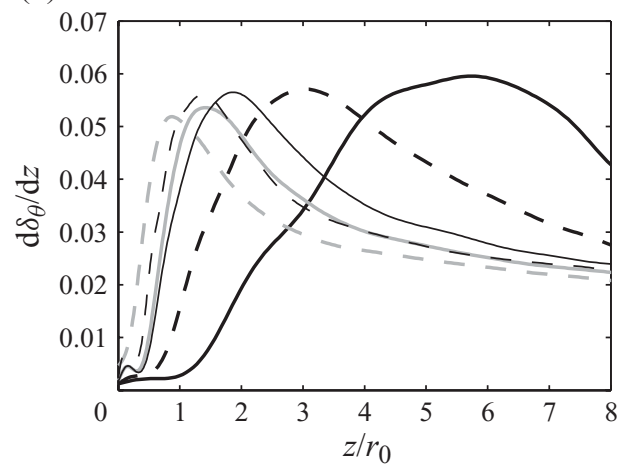

FIGURE 10. Variations of $(a)$ the shear-layer momentum thickness $\delta_{\theta}$ and $(b)$ the spreading rate $\mathrm{d} \delta_{\theta} / \mathrm{d} z$, for - JetD02, - - JetD01, JetD005, - - - JetD0025, JetD005p250 and - - - JetD005p2000.
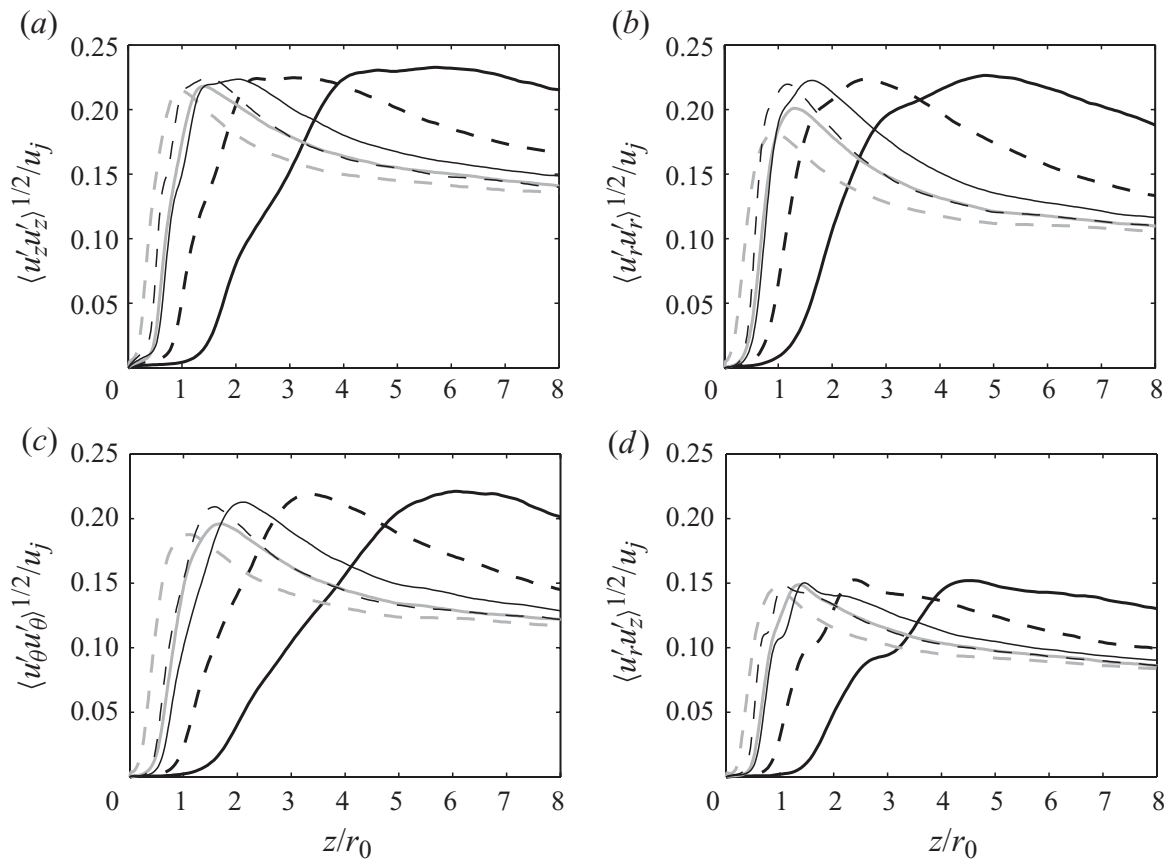

FIGURE 11. Variations along the lip line at $r=r_{0}$ of $(a-c)$ the r.m.s. values of fluctuating velocities $u_{z}^{\prime}, u_{r}^{\prime}$ and $u_{\theta}^{\prime}$ and $(d)$ the magnitude of Reynolds stress $\left\langle u_{r}^{\prime} u_{z}^{\prime}\right\rangle$, for JetD02, - - - JetD01, — JetD005, - - - JetD0025, —— JetD005p250 and - - JetD005p2000.

from Zaman \& Hussain (1980), of a first stage of strong vortex pairings occurring at a location that does not vary much. This may also indicate a feedback mechanism in these four jet simulations without inlet random noise taking place, for instance, at the pipe lip. This feature is however not observed for JetD005p250 and JetD005p2000. Because noise sources in jets can be characterized in terms of the turbulence maxima following Zaman (1986), it is also interesting to notice that the peak levels obtained for velocity $u_{z}^{\prime}$ are all around $0.23 u_{j}$, whereas the peak levels for velocity $u_{r}^{\prime}$ are 


\begin{tabular}{lccccc}
\hline Reference & & & & $\left\langle u_{z}^{\prime 2}\right\rangle^{1 / 2} / u_{j}$ & $\left\langle u_{r}^{\prime 2}\right\rangle^{1 / 2} / u_{j}$ \\
$\max$ at $r=r_{0}$ & $z_{\text {roll }} / r_{0}$ & $z_{\text {pair }} / r_{0}$ & $S t_{\text {pair }}^{\text {peak }}$ & 0.54 & $r_{0}$ \\
JetD02 & 2.5 & 4.8 & 0.54 & 0.233 & 0.226 \\
JetD01 & 1.7 & 2.6 & 0.96 & 0.225 & 0.223 \\
JetD005 & 1.1 & 1.6 & 1.61 & 0.223 & 0.222 \\
JetD0025 & 0.8 & 1.2 & 2.48 & 0.224 & 0.222 \\
JetD005p250 & 0.95 & 1.3 & 1.88 & 0.219 & 0.201 \\
JetD005p2000 & 0.6 & 0.9 & 2.16 & 0.215 & 0.181
\end{tabular}

TABLE 5. Positions $z_{\text {roll }}$ and $z_{\text {pair }}$ at which velocity spectra are calculated along the lip line at $r=r_{0}$, peak Strouhal number in radial velocity spectra at $r=r_{0}$ and $z=z_{\text {pair }}$, and peak r.m.s. values of the axial and radial fluctuating velocities $u_{z}^{\prime}$ and $u_{r}^{\prime}$ along the lip line.
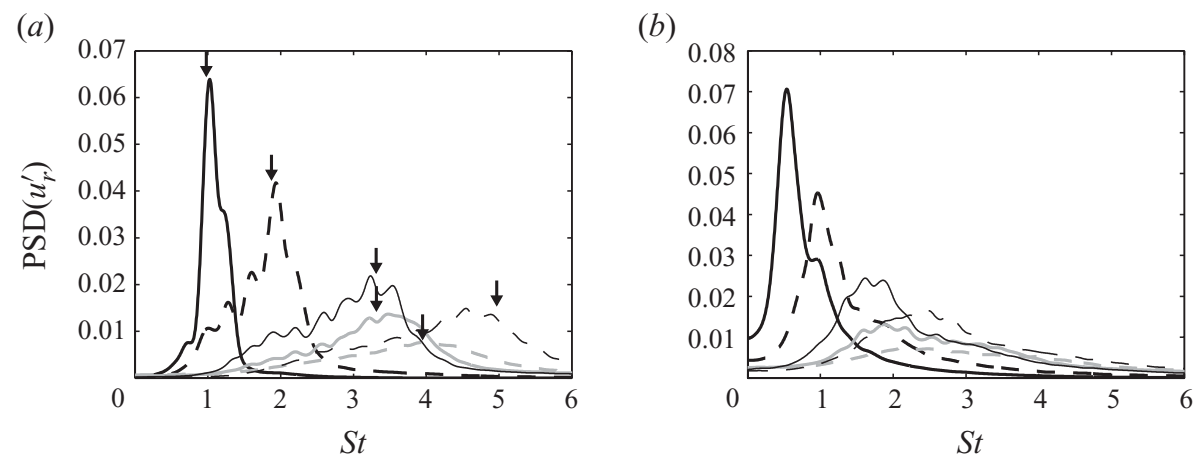

FIGURE 12. Power spectral densities (PSD) of the velocity $u_{r}^{\prime}$ obtained at $r=r_{0}$, as functions of Strouhal number $S t=f D / u_{j}:(a)$ around rolling-up at $z=z_{\text {roll }},(b)$ just downstream of pairing at $z=z_{\text {pair }}$, for JetD02, - - - JetD01, JetD005, - - - JetD0025, JetD005p250 and - - - JetD005p2000 ( $z_{\text {roll }}$ and $z_{\text {pair }}$ are given in table 5). The arrows indicate the frequencies determined from the shear-layer momentum thickness found at $z=0.1 r_{0}$ for JetD005p2000 and at $z=0.4 r_{0}$ for all other jets using $f \delta_{\theta} / u_{j}=0.012$.

around $0.22 u_{j}$ for JetD02, JetD01, JetD005 and JetD0025, but decrease down to $0.18 u_{j}$ for JetD005p2000, as reported in table 6 . These results give evidence of the significant changes that occur in the shear-layer turbulence when transition is initially affected by random disturbances even of very low magnitude, the rolling-up/pairing process being of less importance in this case. This sensitivity to inflow disturbances will be shown to have notable consequences on the acoustic field in $\S 4$.

Spectra of the fluctuating radial velocity $u_{r}^{\prime}$ obtained along the lip line are now presented in figure 12. They are computed at two positions $z_{\text {roll }}$ and $z_{\text {pair }}$ given in table 5, corresponding approximately to the location of the vortex rolling-up and to the end of the first pairing, respectively. More precisely, $z_{\text {pair }}$ is taken at the peak of the r.m.s. fluctuating velocity $u_{r}^{\prime}$ along $r=r_{0}$, and $z_{\text {roll }}$ is near the inflection point that is observed in the graphs of the Reynolds shear stress $\left\langle u_{r}^{\prime} u_{z}^{\prime}\right\rangle$ in figure 11, when possible. Arrows are also plotted in figure 12 to show the frequencies evaluated in the jets using the formula $f \delta_{\theta} / u_{j}=0.012$, from the momentum thicknesses found slightly downstream of the exit section at $z=0.1 r_{0}$ for JetD005p2000 and at $z=0.4 r_{0}$ for all other jets, provided in table 3. In figure $12(a)$, at $z=z_{\text {roll }}$, the velocity spectra are dominated by peaks at $S t=f D / u_{j}$ very close to the values indicated by the 
arrows. The rolling-ups therefore develop at frequencies in agreement with those found experimentally in initially laminar mixing layers, as in the works of Zaman $(1985 a, b)$ for instance. In figure $12(b)$, at $z=z_{p a i r}$, the velocity spectra exhibit peaks at Strouhal numbers $S t_{\text {pair }}^{\text {peak }}$, reported in table 5, which are the first subharmonics of the rolling-up frequencies. This behaviour is observed in all jets. Nevertheless, it is less pronounced in jets with thinner initial shear layers, and when inlet random noise is introduced inside the pipe nozzle, which implies that the rolling-up and pairing process is weaker.

To give a final insight into the development of the shear-layer turbulence, the crosscorrelation functions $\mathscr{R}_{11}^{(\theta)}$ of velocity $u_{z}^{\prime}$ obtained along the lip line are decomposed into their Fourier coefficients with the azimuthal modes $n$, so that

$$
\mathscr{R}_{11}^{(\theta)}(\delta \theta)=\sum_{n=0}^{\infty} a_{n}^{(\theta)} \cos (n \delta \theta)
$$

and coefficient $a_{n}^{(\theta)}$ indicates the relative amplitude of the azimuthal mode $n$.

The axial variations of $a_{0}^{(\theta)}, a_{1}^{(\theta)}, a_{2}^{(\theta)}$ and $a_{3}^{(\theta)}$ from the nozzle lip to slightly downstream of the first vortex pairings are shown in figure 13. At the pipe exit at $z=0$, as previously discussed in $\S 2.3$, the modal distributions vary with the jet conditions. In all jets, the axisymmetric mode $n=0$ is dominant, but its contribution decreases with thinner exit boundary layer and with the introduction of inlet noise. Just downstream of the lip, the relative amplitude of mode $n=0$ rises, implying that the axisymmetric mode grows faster. This behaviour near the jet exit is in agreement with the linear instability analysis conducted by Cohen \& Wygnanski (1987), with the measurements obtained by Raman, Rice \& Reshotko (1994) for an untripped jet at $M=0.2$ and $R e=4 \times 10^{5}$, and with simulation results of Kim \& Choi (2009) for jets at $R e_{D}=10^{5}$. This may be expected, given the experimental findings by Suzuki \& Colonius (2007) that the influence of compressibility on the mode balance of instability waves at the nozzle exit of subsonic round jets is rather small. The relative amplitude of the axisymmetric mode quickly reaches a peak, and then collapses as the contributions of higher modes become stronger due to nonlinear interactions between waves and the generation of three-dimensional turbulence. The modal decomposition of velocity disturbances at the position of vortex rolling-ups, hence the strength of the initial vortex ring, however depends on the jet initial conditions. At rolling-up, mode $n=1$ indeed dominates in the four jets without inlet noise, but its relative amplitude varies from $35 \%$ in JetD02 down to $9 \%$ in JetD0025. The contributions of mode $n=1$ at $z=z_{\text {roll }}$ in the JetD005p250 and JetD005p2000 jets, 5 and $2 \%$ respectively, are still notably lower, which clearly illustrates the weakening of shear-layer vortices when inlet noise is added.

\subsection{Jet flow development}

The effects of the nozzle-exit conditions on the mean flow field of the circular jets are shown in figure 14 with the profiles of the centreline mean axial velocity $u_{c}$ and the jet half-width $\delta_{0.5}$ defined by $\left\langle u_{z}\right\rangle\left(r=\delta_{0.5}\right)=u_{c} / 2$. When the initial momentum thickness $\delta_{\theta}$ is reduced, as suggested by previous results, the jets start to develop on the centreline, and to spread radially, at farther axial distances. This results in longer potential cores, in agreement with the simulations of Kim \& Choi (2009). To quantify the core elongation, the positions $z_{c}$ of the end of the potential core are evaluated in the different jets using $u_{c}\left(z=z_{c}\right)=0.95 u_{j}$. They are given in table 6 and represented in figure 15 as a function of the inlet boundary-layer thickness $\delta$. For the four jets without 


\begin{tabular}{lccc}
\hline & & $\left\langle u_{z}^{\prime 2}\right\rangle^{1 / 2} / u_{j}$ & $\left\langle u_{r}^{\prime 2}\right\rangle^{1 / 2} / u_{j}$ \\
Reference & $z_{c} / r_{0}$ & $\max$ at $r=0$ & 0.139 \\
JetD02 & 8.6 & 0.164 & 0.093 \\
JetD01 & 10.8 & 0.124 & 0.071 \\
JetD005 & 14.1 & 0.109 & 0.057 \\
JetD0025 & 16.8 & 0.105 & 0.071 \\
JetD005p250 & 16.4 & 0.104 & 0.069 \\
JetD005p2000 & 18.1 & 0.101 &
\end{tabular}

TABLE 6. Axial position of the end of the potential core $z_{c}$ and peak r.m.s. values of the axial and radial fluctuating velocities $u_{z}^{\prime}$ and $u_{r}^{\prime}$ along the centreline.

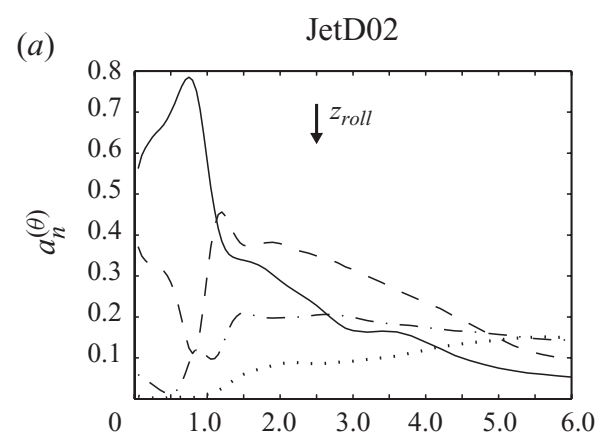

(b)
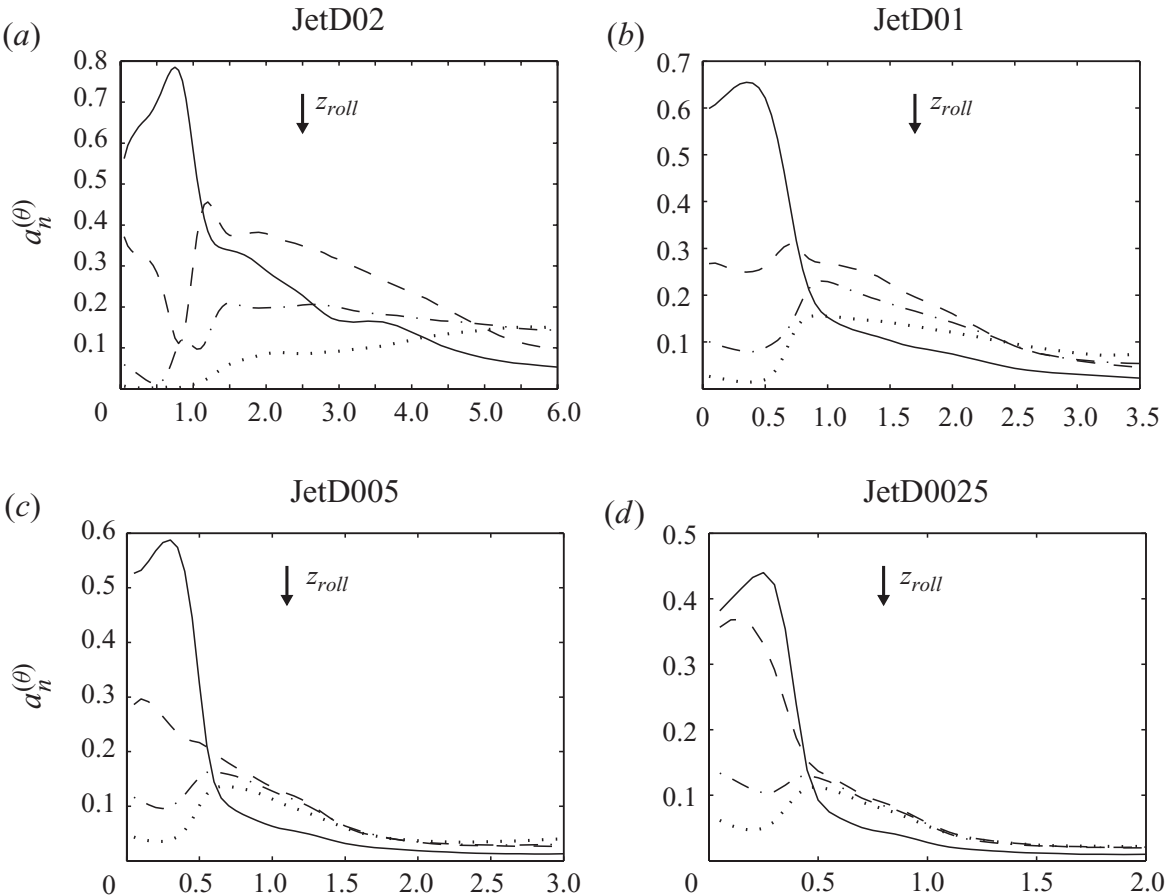

(d)

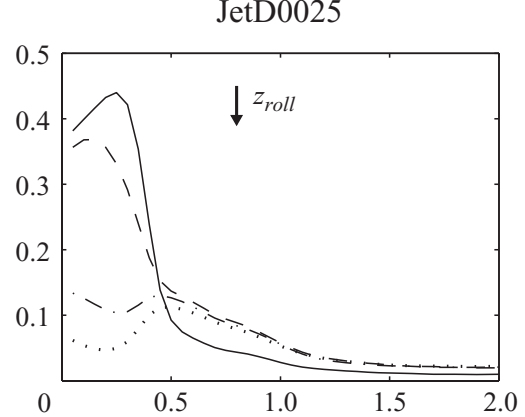

(e)

JetD005p250
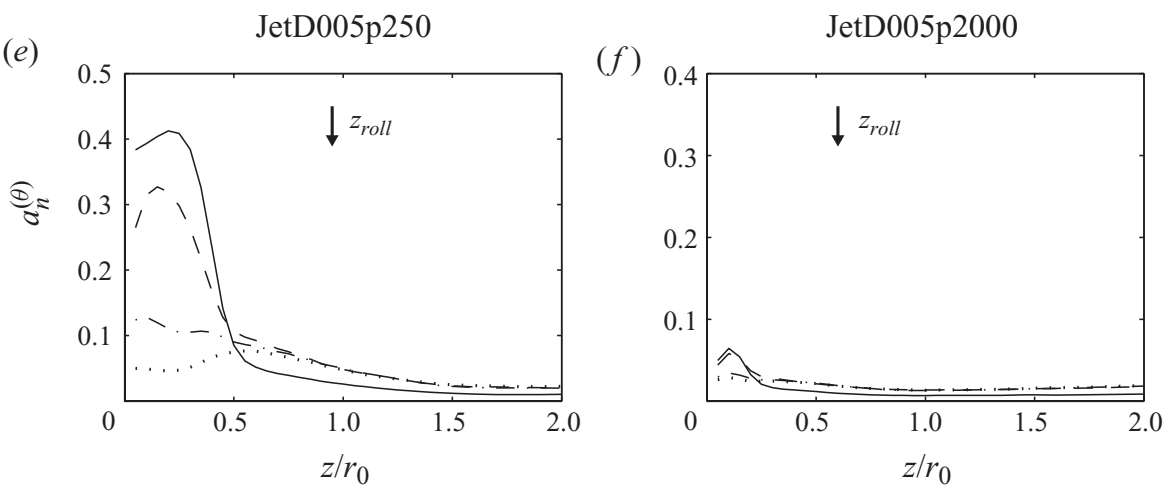

FIGURE 13. $(a-f)$ Variations in the axial direction of the coefficients $a_{n}^{(\theta)}$ obtained from the azimuthal decomposition of cross-correlation functions $\mathscr{R}_{11}^{(\theta)}$ of the fluctuating axial velocity at $r=r_{0}:-n=0,--n=1,-\cdot-\cdot n=2, \cdots \cdots n=3$. The axis limits depend on the jet considered, and $z_{\text {roll }}$ represents the approximated position of vortex rolling-ups provided in table 5. 
(a)

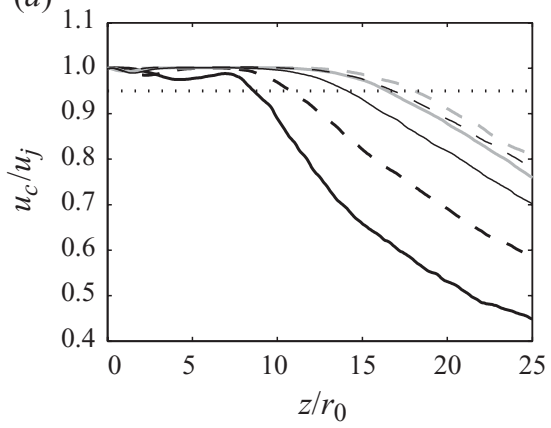

(b)

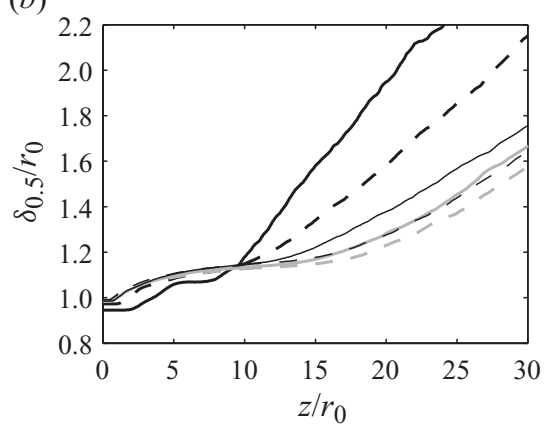

FIGURE 14. Variations of $(a)$ the centreline mean axial velocity $u_{c}$ and $(b)$ the jet half-width $\delta_{0.5}$, for - JetD02, - - - JetD01, — JetD005, - - JetD0025, — JetD005p250 and --- JetD005p2000 (the dotted line represents $u_{c}=0.95 u_{j}$ ).

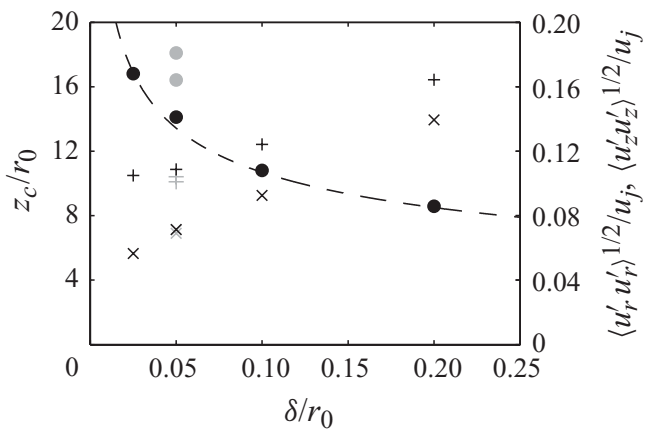

FIGURE 15. Variations with inlet boundary-layer thickness $\delta$. Symbols: $\bullet$, position of the end of the potential core $z_{c}$; + and $\times$, peak r.m.s. values of velocities $u_{z}^{\prime}$ and $u_{r}^{\prime}$ along the centreline. Black symbols: jets without inlet noise; grey symbols: jets with inlet noise; - - $z_{c} / r_{0}=5 /\left(\delta / r_{0}\right)^{1 / 3}$.

inlet noise, they range from $z_{c}=8.6 r_{0}$ for JetD02 up to $z_{c}=16.8 r_{0}$ for JetD0025, and appear to be well predicted by the equation $z_{c} / r_{0}=5 /\left(\delta / r_{0}\right)^{1 / 3}$. The values obtained for JetD005p250 and JetD005p2000 using inlet noise are $z_{c}=16.4 r_{0}$ and $z_{c}=18.1 r_{0}$, respectively. They are larger than the value $z_{c}=14.1 r_{0}$ found for JetD005, which indicates that the potential core length increases with the magnitude of the random disturbances. Similar lengthening of the jet core was observed experimentally by Raman et al. $(1989,1994)$ when tripping the exit boundary layer of jets at Reynolds numbers around $5 \times 10^{5}$. The jet mean flow therefore develops more slowly when the exit $\delta_{\theta}$ becomes smaller, or when random disturbances are added in the pipe.

To characterize the magnitude of the jet turbulence, the levels of the fluctuating axial and radial velocities along the centreline are presented in figure 16. As the initial shear-layer thickness decreases, they reach peaks with lower amplitude, farther in the downstream direction, both for the axial velocity $u_{z}^{\prime}$ as in Kim \& Choi (2009) and for the radial velocity $u_{r}^{\prime}$. The peak values are reported in table 6 and shown in figure 15 as functions of $\delta$. The maxima of the centreline r.m.s. levels for $u_{r}^{\prime}$ are for instance $0.134 u_{j}$ for JetD02, but only $0.057 u_{j}$ for JetD0025. This trend could be due to the apparently contradictory results of getting earlier shear-layer development but also longer potential core when $\delta_{\theta}$ becomes smaller at the nozzle exit. The shear-layer transition, and consequently its related peak r.m.s. velocity levels around 
(a)

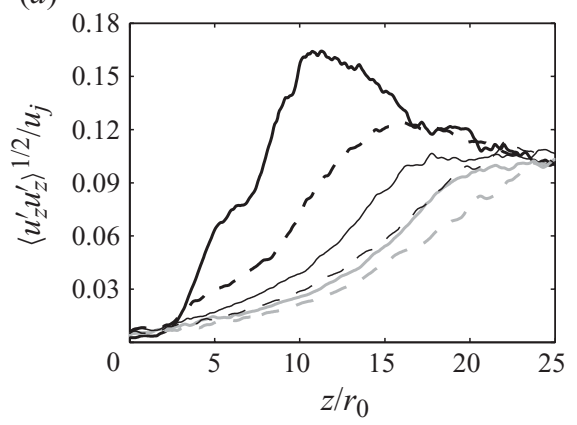

(b)

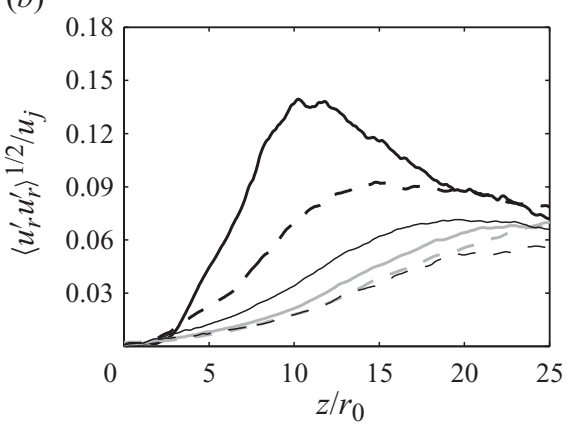

FIGURE 16. $(a, b)$ Variations along the centreline at $r=0$ of the r.m.s. values of fluctuating velocities $u_{z}^{\prime}$ and $u_{r}^{\prime}$, for $\longrightarrow$ JetD02, - - JetD01, JetD005, - - JetD0025, JetD005p250 and --- JetD005p2000.

$0.2 u_{j}$ observed in figure 11 , occurs farther from the end of the potential core where the shear-layer vortical structures merge on the jet axis, which leads to lower centreline turbulence levels. Regarding the influence of adding inflow noise, it appears rather weak because the peaks of the fluctuation levels for JetD005p250 and JetD005p2000 are very similar to those for JetD005 with same initial $\delta_{\theta}$.

The variations of the integral length scales associated with axial and radial velocities $u_{z}^{\prime}$ and $u_{z}^{\prime}$ are finally considered. These scales are computed respectively as

$$
L_{11}^{(1)}=\int_{0}^{\delta z_{11}^{0.02}} \mathscr{R}_{11}^{(1)} \mathrm{d}(\delta z) \quad \text { and } \quad L_{22}^{(1)}=\int_{0}^{\delta z_{22}^{0.02}} \mathscr{R}_{22}^{(1)} \mathrm{d}(\delta z)
$$

from the cross-correlation functions determined at $(r, \theta, z)$ by

$$
\mathscr{R}_{11}^{(1)}(\delta z)=\frac{\left\langle u_{z}^{\prime}(r, \theta, z) u_{z}^{\prime}(r, \theta, z+\delta z)\right\rangle}{\left\langle u_{z}^{\prime 2}(r, \theta, z)\right\rangle^{1 / 2}\left\langle u_{z}^{\prime 2}(r, \theta, z+\delta z)\right\rangle^{1 / 2}}
$$

and

$$
\mathscr{R}_{22}^{(1)}(\delta z)=\frac{\left\langle u_{r}^{\prime}(r, \theta, z) u_{r}^{\prime}(r, \theta, z+\delta z)\right\rangle}{\left\langle u_{r}^{\prime 2}(r, \theta, z)\right\rangle^{1 / 2}\left\langle u_{r}^{\prime 2}(r, \theta, z+\delta z)\right\rangle^{1 / 2}},
$$

where $\delta z$ is the axial separation. In the calculation of the length scales, to avoid possible artefacts coming from non-perfectly converged correlations, integrand limits $\delta z_{11}^{0.02}$ and $\delta z_{22}^{0.02}$ are imposed. They are chosen so that $\mathscr{R}_{11}^{(1)}\left(\delta z_{11}^{0.02}\right)=0.02$ and $\mathscr{R}_{22}^{(1)}\left(\delta z_{22}^{0.02}\right)=0.02$.

The integral length scales obtained along the lip line in the different jets are shown in figure 17. For both axial and radial velocities, the length scales grow more slowly with thinner initial boundary layer and inlet noise.

\section{Far-field results}

This section presents the effects of the jet initial conditions on noise, by showing pressure snapshots, and the features of the acoustic far fields. The simulation results are also compared to experimental data obtained for initially laminar jets at moderate Reynolds numbers as well as for jets at Mach number 0.9 and high Reynolds numbers.

\subsection{Pressure snapshots}

The near acoustic fields issued from the LES are propagated to 60 radii from the nozzle exit, by solving the linear acoustical equations as described in $\S 2.4$. The wave 

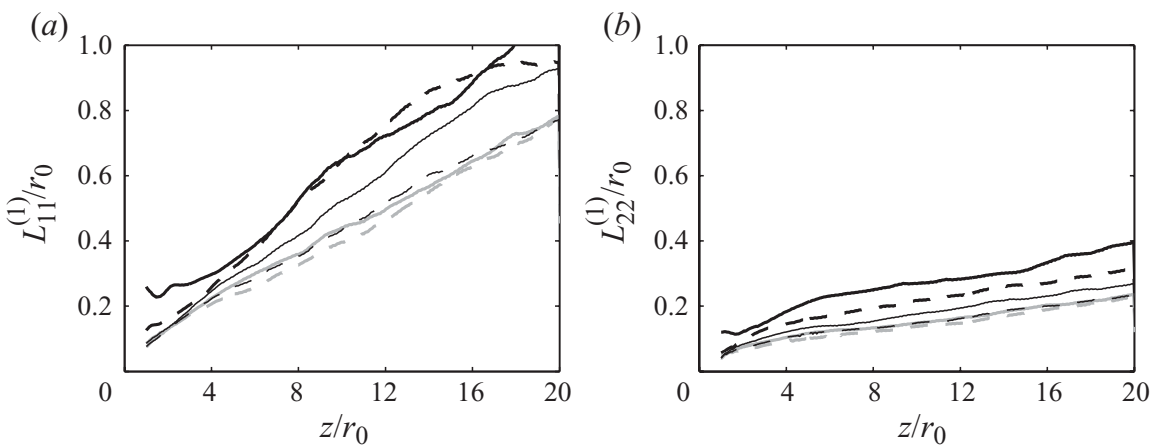

FIGURE 17. $(a, b)$ Variations in the axial direction of the integral length scales $L_{11}^{(1)}$ and $L_{22}^{(1)}$ calculated from velocities $u_{z}^{\prime}$ and $u_{r}^{\prime}$ along $r=r_{0}$, for - JetD02, -- JetD01, JetD005, - - - JetD0025, — JetD005p250 and - - JetD005p2000.

(a)

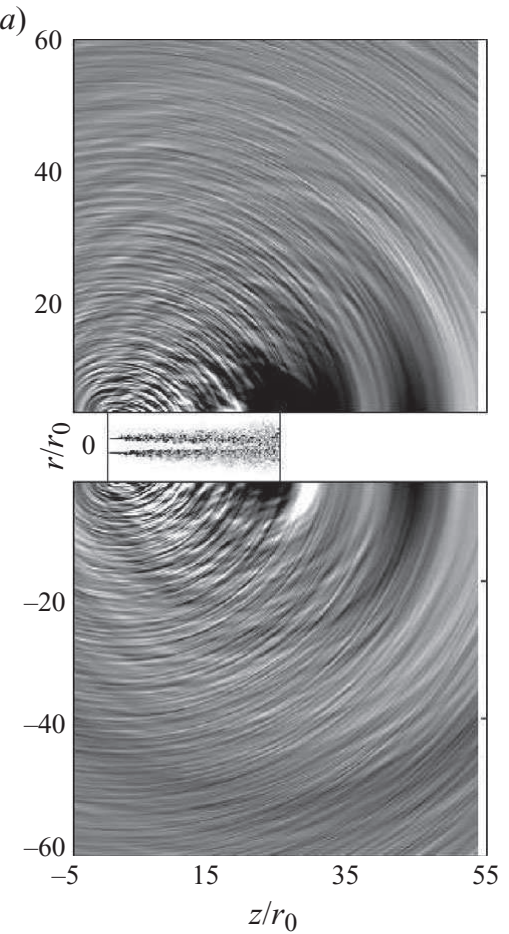

(b)

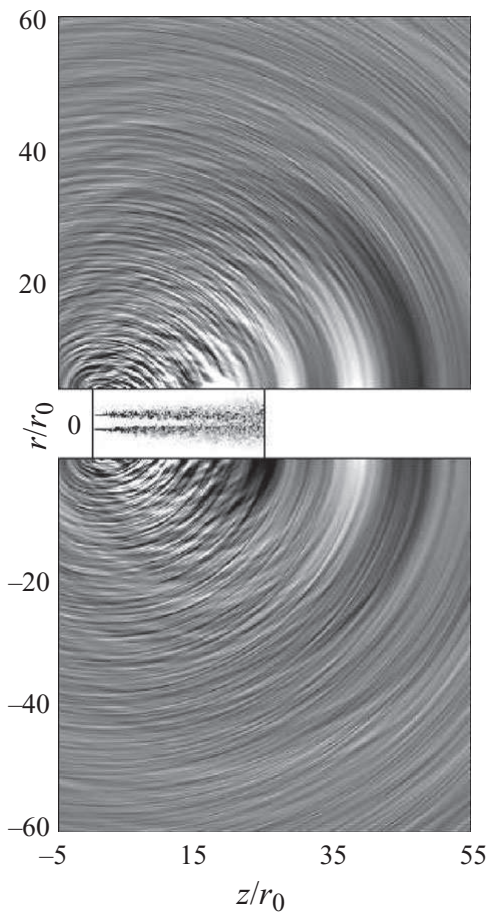

FigURE 18. Snapshots in the $(z, r)$ plane of the pressure field obtained from the acoustical simulations for JetD005p2000, using $(a)$ the full control surface and $(b)$ a control surface limited to $z \leqslant z_{c}+2 r_{0}$, where $z_{c}$ is the position of the potential core end. The colour scales range from -40 to $40 \mathrm{~Pa}$ for pressure and from 0 to $8 u_{j} / r_{0}$ for vorticity norm in the LES jet flow, from white to black.

extrapolation is performed from a cylindrical control surface located at $r=5.25 r_{0}$, extending from $z=-0.6 r_{0}$ to $z=25 r_{0}$ in the axial direction. A snapshot of the pressure field thus determined for JetD005p2000 is provided in figure 18. The propagation of the sound waves generated in the turbulent shear layers seems to be properly taken into account. Unfortunately, low-frequency waves of high magnitude originating from the end of the control surface are also observed. Based on the far-field extrapolations presented in Appendix $\mathrm{C}$ using control surfaces shortened in the axial direction or 


\begin{tabular}{lcccc}
\hline Reference & $M$ & $R e_{D} \times 10^{-5}$ & $\delta_{\theta} / r_{0} \times 10^{3}$ & $u_{z}^{\prime} / u_{j}$ \\
Zaman $(1985 a, b)$ & $0.12 \rightarrow 0.23$ & $0.7 \rightarrow 1.3$ & $7.9 \rightarrow 5.7$ & $\leqslant 0.01$
\end{tabular}

TABLE 7. Experiments on initially laminar jets by Zaman $(1985 a, b)$ : Mach and Reynolds numbers, initial shear-layer momentum thickness and maximum intensity of the velocity $u_{z}^{\prime}$ at the nozzle exit. The arrow indicates the parameter variations.

(a)

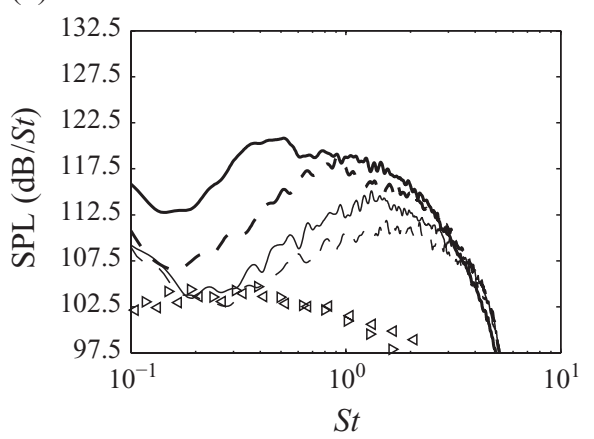

(b)

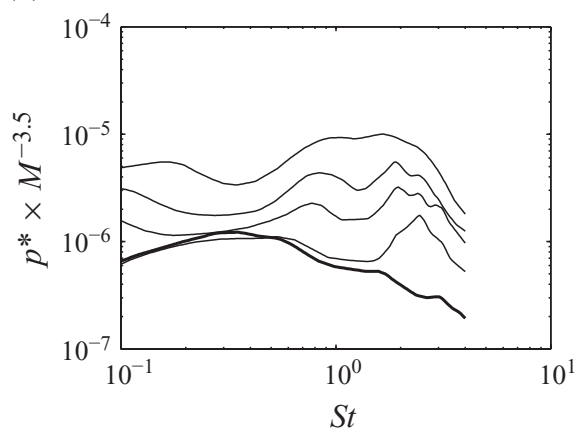

FigURE 19. Narrow-band far-field sound pressure levels (SPL) at a radiation angle of $90^{\circ}$, as functions of Strouhal number $S t=f D / u_{j}:(a) \longrightarrow$ JetD02, - - - JetD01, $\longrightarrow$ JetD005,

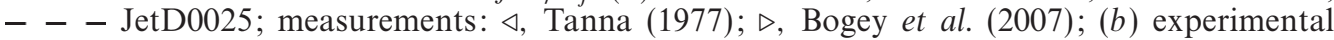
data by Zaman $(1985 a, b)$ for untripped jets $(-)$ with exit boundary-layers momentum thickness $\delta_{\theta} / r_{0} \times 10^{3}=7.9,7.3,6.5,5.7$ (from top to bottom) and a tripped jet (—).

located farther from the centreline, these spurious waves appear to be generated because the downstream part of the control surface is too close to the aerodynamic flow field. This led us to carry out for all jets wave extrapolations using control surfaces limited to $z=z_{c}+2 r_{0}$ in the axial direction, where $z_{c}$ is the axial location of the end of the potential core given in table 6. In this way, as illustrated in the pressure snapshots of figure 18, the low-frequency unphysical waves are weakened without appreciably affecting the waves emitted by the jets in the sideline direction. This point is supported by pressure spectra, as those reported in figure 25 at the radiation angle of $90^{\circ}$ for JetD02. The sound spectra at radiation angles of 60 and $90^{\circ}$ and the azimuthal cross-correlations of the fluctuating pressure at $90^{\circ}$, which will be presented in $\S 4.3$, are estimated from these shortened surfaces, whereas the other noise features are obtained from the full control surface. Note finally that the influence of the radial location and the limited axial extend of the extrapolation surface is probably weak on sound spectra even for shallow radiation angles; see for instance figure 25 for the spectra computed at $40^{\circ}$ for JetD02.

\subsection{Comparison with measurements}

A qualitative comparison of the sound fields computed for the fully initially laminar jets JetD02, JetD01, JetD005 and JetD0025 is made with some experimental results obtained by Zaman $(1985 a, b)$. As shown in table 7 , this author indeed considered untripped jets at $R e_{D} \leqslant 2.5 \times 10^{5}$ characterized by nozzle-exit turbulent intensities lower than $1 \%$, and boundary-layer momentum thicknesses decreasing from $\delta_{\theta}=0.0079 r_{0}$ down to $\delta_{\theta}=0.0057 r_{0}$ with the Mach number.

Far-field pressure spectra calculated for JetD02, JetD01, JetD005 and JetD0025 at the radiation angle of $90^{\circ}$ are represented in figure 19(a), while corresponding spectra provided by Zaman $(1985 a, b)$ for four untripped jets are displayed in figure 19(b). A 


$\begin{array}{llc}\text { Reference } & M & R e_{D} \times 10^{-5} \\ \text { Mollo-Christensen, Kolpin \& Martucelli (1964) } & 0.9 & 5.4 \\ \text { Lush (1971) } & 0.88 & 5 \\ \text { Tanna (1977) } & 0.9 & 10 \\ \text { Bogey et al. (2007) } & 0.9 & 7.8\end{array}$

TABLE 8. Experiments on jets at $M \simeq 0.9$ and $R e_{D} \geqslant 5 \times 10^{5}$ : Mach and Reynolds numbers.

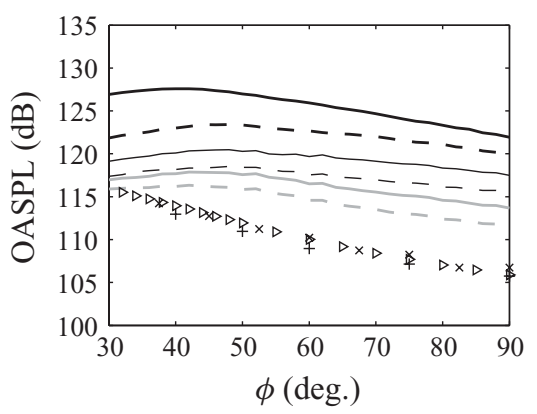

Figure 20. Overall sound pressure levels (OASPL) obtained at $60 r_{0}$ from the jet nozzle exit, as functions of the radiation angle relative to the jet direction, for - JetD02, - - JetD01, — JetD005, - - - JetD0025, — JetD005p250 and - - - JetD005p2000. Measurements: +, Mollo-Christensen et al. (1964); ×, Lush (1971); $\triangleright$, Bogey et al. (2007).

strong resemblance can be seen between the simulation and the experimental results. The acoustic spectra similarly exhibit important additional bumps with respect to spectra obtained for $M=0.9$ jets at Reynolds numbers around $10^{6}$ in figure $19(a)$, and for a tripped jet in figure 19(b). In both cases, the amplitude of these noise components moreover decreases and their peak frequency becomes higher with a thinner initial shear layer. More quantitatively, the additional peaks in Zaman's spectra, which are found for Strouhal numbers between 1.5 and 2 depending on the exit boundarylayer momentum thickness, roughly correspond to frequencies satisfying the relation $f \delta_{\theta} / u_{j}=0.006$, which is around natural vortex-pairing frequency in laminar shear layers. A similar link will be exhibited in the next section for the sound spectra determined from the computations. A good agreement with experiments is thus observed, which supports that the simulations correctly predict noise generation mechanisms in the present initially laminar jets.

\subsection{Sound field properties}

The acoustic far fields determined from the extrapolation of the LES near fields are now described in detail. The sound pressure levels calculated at $60 r_{0}$ from the jet nozzle exit, for $S t \geqslant 0.1$, are first represented in figure 20. Compared with measurements provided by Mollo-Christensen et al. (1964), Lush (1971) and Bogey et al. (2007) for jets at $M \simeq 0.9$ and $R e_{D} \geqslant 5 \times 10^{5}$ listed in table 8 , they are strongly higher, which indicates the presence of additional noise components. This corresponds accurately to the observations made by Zaman (1985a,b) and Bridges \& Hussain (1987) concerning the noise radiated by untripped jets at $R e_{D}<5 \times 10^{5}$. In figure 20, a significant decrease in the acoustic levels when just adding inlet random disturbances of low amplitude in the pipe is also noticed. Furthermore, it can be pointed out that the levels obtained for the jets are much more scattered at an emission angle of $90^{\circ}$ than at $30^{\circ}$. At the latter angle, for the jets with thin initial shear layers, they are even 


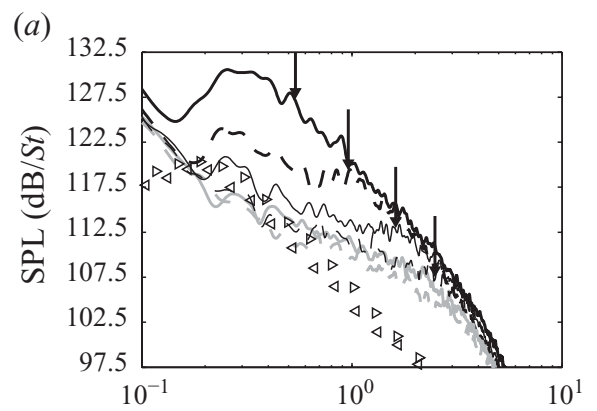

(b)
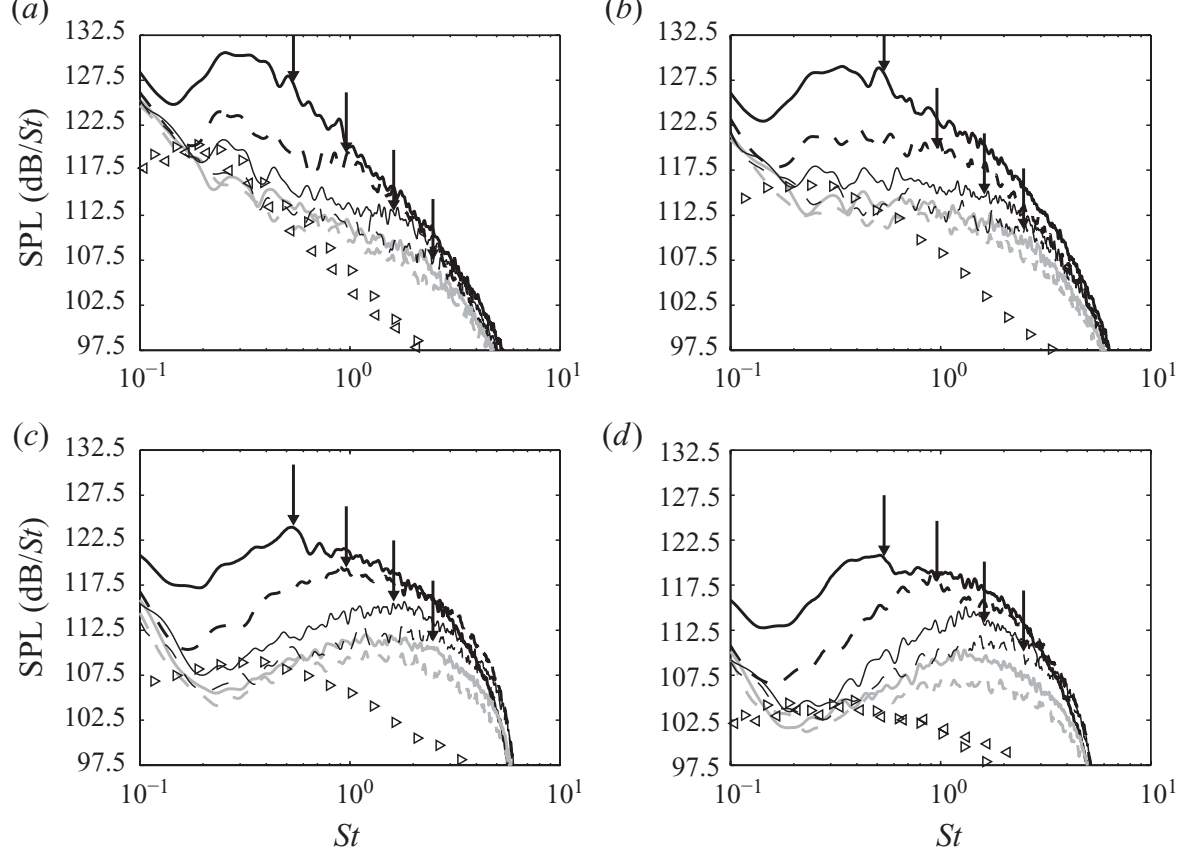

FIGURE 21. Narrow-band sound pressure levels (SPL) obtained at $60 r_{0}$ from the jet nozzle exit, as functions of Strouhal number $S t=f D / u_{j}$, for radiation angles relative to the jet direction of $(a)$ 30, (b) 40, (c) 60 and $(d) 90^{\circ}$, for - JetD02, - - - JetD01, JetD005, - - JetD0025, JetD005p250 and - - JetD005p2000. The arrows indicate the vortex-pairing Strouhal numbers $S t_{\text {pair }}^{\text {peak }}$ in JetD02, JetD01, JetD005 and JetD0025. Narrow-band measurements: $\triangleleft$, Tanna (1977); $\triangleright$, Bogey et al. (2007).

rather close to the experimental data. The sensitivity of the sound fields to the jet exit conditions is therefore higher in the transverse direction than in the downstream direction.

To study the influence of the inflow conditions on the jet sound sources, the pressure spectra obtained at 60 radii from the pipe exit for angles of 30, 40, 60 and $90^{\circ}$ relative to the jet direction are represented in figure 21. Spurious low-frequency components, certainly coming from the downstream part of the control surface used for the far-field extrapolation as discussed in $\$ 4.1$. and Appendix C, are noted for Strouhal numbers lower than 0.15 for all angles. More interestingly, compared with the experimental sound spectra of Tanna (1977) and Bogey et al. (2007) for Mach number 0.9 jets at Reynolds numbers $R e_{D} \geqslant 7.8 \times 10^{5}$, the spectra contain additional high-frequency bumps. This is in good agreement with the experimental works conducted by Zaman (1985a,b) (see figure 19b) and Bridges \& Hussain (1987) to investigate noise generation in tripped and untripped jets at $\operatorname{Re}_{D}<5 \times 10^{5}$. Accordingly, the simulated jets generate sound fields typical of untripped jets at moderate Reynolds numbers, whereas the jets of Tanna (1977) and Bogey et al. (2007), which are very likely to contain significant initial turbulence levels due to their high Reynolds numbers, radiate noise in the same way as tripped jets. In figure 21 , the extra noise components are in addition characterized by peak frequencies corresponding well to the Strouhal numbers $S t_{\text {pair }}^{\text {peak }}$ of the first vortex pairings in the mixing layers given in table 5, indicated by arrows for JetD02, JetD01, JetD005 and JetD0025. 
The present results hence show additional acoustic radiation due to the first stage of pairing of shear-layer coherent vortices in initially laminar jets, as previously in Zaman (1985a,b) and Bridges \& Hussain (1987).

In the four jets without inlet noise, when the nozzle-exit boundary-layer thickness becomes smaller, the pairing noise moves to higher frequencies, as expected. Thus the low-frequency part of the spectra is less and less affected by the pairing noise, and gets closer to the experimental data for high-Reynolds-number jets. This trend is clearly visible for example in the spectra at $90^{\circ}$ in which the sound levels for $0.15 \leqslant S t \leqslant 0.4$ from JetD005 and JetD0025 are similar to the measurements. As the initial $\delta_{\theta}$ is reduced in the simulations, the levels of the pairing noise also decrease. This could be connected to the fact that in jets with thinner shear layers the azimuthal correlations of velocity fluctuations just downstream of the exit section are lower, as evidenced in table 3 and in figures 3 and 13, thus leading to weaker vortex pairings and less efficient noise generation.

The contribution of vortex pairings to the far-field noise appears to depend on the radiation angle. It is in particular higher at $90^{\circ}$ than at $30^{\circ}$. In JetD005 for instance, at $90^{\circ}$, the peak associated with pairing noise is $10 \mathrm{~dB}$ above the maxima reached by the spectra for high-Reynolds-number jets, whereas at $30^{\circ}$, the pairing noise, albeit still very distinctively visible around $S t_{\text {pair }}^{\text {peak }}=1.61$, does not exceed the component at $S t \simeq 0.2$. This could be expected because in subsonic jets, without pairing noise, the acoustic component dominating in the downstream direction is much higher than the sideline acoustic component.

It is further interesting to remark that, in the jet direction, not only do vortex pairings result in extra noise but they can also modify the magnitude of the predominant component at $S t \simeq 0.2$. An amplification of this component is specially observed in the JetD01 and JetD02 jets with thick nozzle-exit boundary layers. In this case, the source responsible for the downstream noise component, associated with the mixing-layer merging on the centreline at the end of the jet core in Bogey \& Bailly (2007), is then strengthened by the pairings. This may be related to the fact that, as the initial shear layer becomes thicker, pairings take place closer to the end of the potential core. In this way, turbulent intensities at $r=0$ are higher, their peak values increasing for instance from $10.5 \%$ in JetD0025 to $16.4 \%$ in JetD02 as shown in figure 16 and in table 6, which gives rise to stronger sound sources on the centreline.

Finally, the addition of inlet random disturbances inside the pipe nozzle is observed to significantly alter the far-field sound spectra, and more precisely to lessen the bumps associated with vortex-pairing noise. With respect to JetD005 without inlet noise, the peaks in the pressure spectra evaluated at $90^{\circ}$ are then reduced by $5 \mathrm{~dB}$ in JetD005p250, and by $8 \mathrm{~dB}$ in JetD005p2000. The lowering of the pairing noise in these two jets is not surprising, because of the weakening of the rolling-up/pairing process noticed in $\S 3.3$. Such variations in the acoustic field are however spectacular and rather unexpected given the jet initial conditions. For JetD005 and JetD005p250, compare for instance the minute differences in table 3 between the exit conditions, namely same boundary-layer momentum thicknesses and r.m.s. velocity fluctuations but slightly lower azimuthal velocity correlations in JetD005p250, with the $5 \mathrm{~dB}$ reduction in the sideline sound spectra. It can also be noted that the noise components below $S t=0.4$ do not change much with the inlet forcing, probably because of the similarities of the flow fields of JetD005, JetD005p250 and JetD005p2000 downstream of the vortex pairings; refer to $\$ 3.4$. For noise reduction purposes, this suggests that modifying jet inflow conditions may strongly impact on sound sources associated with the early shear-layer development, while leaving other sources relatively unaffected. 


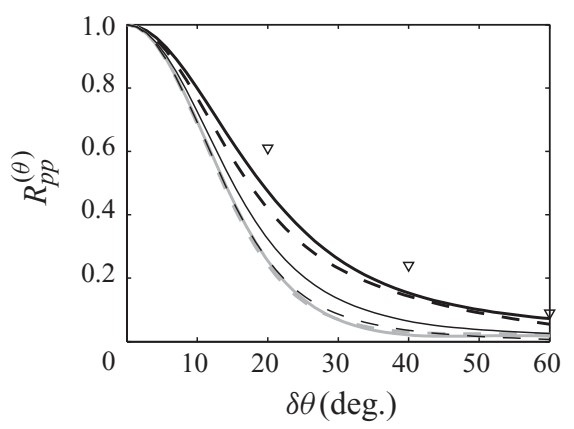

FIGURE 22. Azimuthal cross-correlations of the fluctuating pressure high-pass filtered for $S t \geqslant 0.1$, at $60 r_{0}$ from the jet exit at $90^{\circ}$ relative to the jet direction, for - JetD02, - _ _ JetD01, — JetD005, - - - JetD0025, J JetD005p250 and - - - JetD005p2000. Measurements: $\nabla$, Maestrello $(1976)\left(M=0.7, R_{D}=4.3 \times 10^{5}\right)$.

The spatial structure of the acoustic fields generated by the jets is finally explored by computing azimuthal cross-correlation functions $\mathscr{R}_{p p}^{(\theta)}$ in the following way, at point $(r, \theta, z)$ :

$$
\mathscr{R}_{p p}^{(\theta)}(\delta \theta)=\frac{\left\langle p^{\prime}(r, \theta, z) p^{\prime}(r, \theta+\delta \theta, z)\right\rangle}{\left\langle p^{\prime 2}(r, \theta, z)\right\rangle^{1 / 2}\left\langle p^{\prime 2}(r, \theta+\delta \theta, z)\right\rangle^{1 / 2}} .
$$

Because of the spurious low-frequency waves observed in the sound spectra, they are calculated from the far-field fluctuating pressure signals $p^{\prime}$ in which the components $S t \leqslant 0.1$ are removed by a high-pass filtering. The influence on the correlations might be notable at small radiation angles, at which low-frequency noise dominates, but should be rather weak in the sideline direction. At $90^{\circ}$ relative to the jet direction for example, in figure 21 , the strongest contributions to the sound spectra are noticed for Strouhal numbers higher than 0.2 .

The correlation functions obtained in the jets are therefore presented in figure 22 for the angle of $90^{\circ}$. Compared with the sideline correlations provided by Maestrello (1976) for a high-Reynolds-number jet at $M=0.7$, they are close but slightly lower. This may be due to Mach number effects, as suggested by experimental data of Juvé \& Sunyach (1978) and simulation results of Bogey \& Bailly (2006a). In the present jets, the azimuthal correlations decrease when thinner initial boundary layer is specified. For a separation distance of $\delta \theta=30^{\circ}$, the correlations are for instance around 0.3 for JetD02 but 0.1 for JetD0025. The azimuthal correlations obtained from the two jets with inlet noise, JetD005p250 and JetD005p2000, nearly collapse, and are lower than those for JetD005. The variations of the azimuthal pressure cross-correlations are thus similar to those displayed by the azimuthal velocity correlations in the developing shear layers in figures 3 and 13.

\section{Conclusion}

The LES of round jets at $M=0.9$ and $R e_{D}=10^{5}$ presented in this paper show the significant effects of the nozzle-exit boundary-layer thickness and turbulence conditions on the aerodynamic development and the acoustic field of initially laminar subsonic jets. The numerical results also agree well with measurements obtained in studies dealing separately with axisymmetric shear layers, jets or subsonic jet noise. In this way, the present simulations are used as numerical experiments under controlled conditions to reproduce and complement the experimental findings. 
Decreasing the nozzle-exit boundary-layer momentum thickness in initially laminar jets is found to especially affect the flow development. It leads in particular to an elongation of the potential core and to a reduction of centreline turbulence intensities. It does not seem however sufficient to get the shear-layer development and the acoustic fields that are experimentally observed for turbulent jets at $R e_{D} \geqslant 5 \times 10^{5}$, namely for practical jets. Coherent vortex pairings and their strong generated noise are indeed noticed in the present initially laminar jets, whatever the exit momentum thickness may be.

The early turbulent development in initially laminar jets is dominated by processes of vortex rolling-up and pairing, which occur at frequencies related to the initial shear-layer thickness, but whose other characteristics including azimuthal properties and strength mainly depend on initial turbulence. Therefore, it turns out to be important in jet simulations to control the initial velocity disturbances, so as to impose nozzle-exit conditions as close as possible to the experimental conditions, when they are known. In the present LES, the jet shear-layer transition is for example modified appreciably by the addition of small inlet noise, leading to a weakening of the vortex pairings, to an increase of the potential core length, and to a spectacular lowering of the far-field sound pressure levels. Such changes probably arise even for minute differences in the inflow conditions, as can be seen from the results obtained for JetD005 and JetD005p250, which are two initially fully laminar jets with same nozzle-exit boundary-layer thickness and turbulent intensities.

Finally, the present results illustrate the importance of taking into account the issue of jet inflow conditions in simulations, as well as in experiments as recently pointed out by Kearney-Fischer, Kim \& Samimy (2009). This should be particularly the case in studies dealing with the prediction and the reduction of jet noise. For instance, if one aims to investigate the effects of devices such as chevrons, tabs or microjets, one should ensure that the uncertainties due to the inflow conditions are lower than the variations of the sound pressure levels around $-3 \mathrm{~dB}$ expected according to experiments such as those by Saiyed, Mikkelsen \& Bridges (2003), Callender, Gutmark \& Martens (2005), Alkislar, Krothapalli \& Butler (2007) and Castelain et al. (2008).

This work was granted access to the HPC resources of the Institut du Développement et des Ressources en Informatique Scientifique (IDRIS-CNRS) under the allocation 2009-020204 made by GENCI (Grand Equipement National de Calcul Intensif). The authors are especially grateful to Jean-Michel Dupays from IDRIS for his technical assistance. They would also like to thank Dr Khairul Zaman for his insightful remarks on the present work.

Supplementary movies are available at journals.cambridge.org/flm.

\section{Appendix A. An explicit non-centred selective filter}

In this appendix, the coefficients used for a non-centred filter are given. To improve the stability of the jet LES around the nozzle lips, the non-centred filter proposed by Berland et al. (2007b) to damp out grid-to-grid oscillations at the second row of points above boundaries has indeed been replaced by another filter with similar properties in the wavenumber space. Using this filter, a function $f$ discretized on a 


$$
\begin{aligned}
& d_{-2}^{28}=0.0307159855992469 \\
& d_{-1}^{28}=-0.148395705486028 \\
& d_{0}^{28}=0.312055385963757 \\
& d_{1}^{28}=-0.363202245195514 \\
& d_{2}^{28}=0.230145457063431 \\
& d_{3}^{28}=-0.0412316564605079 \\
& d_{4}^{28}=-0.0531024700805787 \\
& d_{5}^{28}=0.0494343261171287 \\
& d_{6}^{28}=-0.0198143585458560 \\
& d_{7}^{28}=0.00339528102492129 \\
& d_{8}^{28}=0
\end{aligned}
$$

TABLE 9. Coefficients $d_{j}^{28}$ of an explicit non-centred selective filter.

uniform grid $\left(x_{i}\right)$ is filtered to provide the quantity

$$
\tilde{f}\left(x_{i}\right)=f\left(x_{i}\right)-\sigma \sum_{j=-2}^{8} d_{j}^{28} f\left(x_{i}+j \Delta x\right),
$$

where $\Delta x$ is the mesh spacing, $\sigma$ is the filtering strength between 0 and 1 , and $d_{j}^{28}$ are the coefficients of the filter reported in table 9 .

\section{Appendix B. Effects of grid parameters on jet flow features}

In this appendix, the effects of the grid parameters on the present initially laminar jets are examined. Two additional jet simulations, JetD02Lz20 and JetD02grid, have been performed. The jet conditions in both cases are those of the JetD02 jet defined in $\S 2.1$. The thickness of the exit boundary layer is thus $\delta=0.2 r_{0}$, and there is no inlet random noise. The mesh grids are however modified to check the influence of the sponge zone and the grid resolution. In JetD02Lz20, the grid is the same as the grid in JetD02, but it is shortened by $5 r_{0}$ in the axial direction so that the sponge zone begins at $z=20.5 r_{0}$ instead of $z=25.5 r_{0}$. In JetD02grid, the finer grid specified in $\S 2.2$ for the JetD01 jet is used, shortened by $5 r_{0}$ in the axial direction as previously. The axial and radial mesh sizes at the pipe lip are then half those in JetD02 and JetD02Lz20, and $\Delta r$ is equal to $\delta / 14$ instead of $\delta / 7$ in the inlet boundary layer and the shear layer. The simulation times for JetD02Lz20 and JetD02grid are finally around $170 D / u_{j}$.

The developments of the mixing layers in JetD02, JetD02Lz20 and JetD02grid are first compared by showing in figure 23 the variations of the shear-layer momentum thickness and the r.m.s. axial velocity fluctuations at $r=r_{0}$. The results from JetD02 and JetD02Lz20 are quite similar, especially for the turbulent intensities. They also agree well with the results obtained from JetD02grid using a finer grid. The only notable difference in this case is a slight decrease of the r.m.s. velocity fluctuations downstream of $z \simeq 4 r_{0}$. From the pipe-nozzle exit to the maxima of turbulent intensities, the curves are however superimposed, which supports that the laminarturbulent transitions in the shear layers are the same, regardless of the grid.

The jet flow features from JetD02, JetD02Lz20 and JetD02grid are now characterized by plotting in figure 24 the centreline variations of the mean values and the r.m.s. fluctuating values of the axial velocity. The developments of the JetD02 and JetD02Lz20 jets are very similar, whereas that of the JetD02grid jet seems to 
(a)

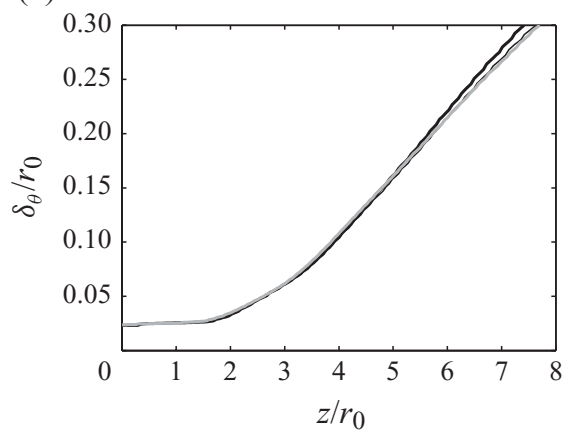

(b)

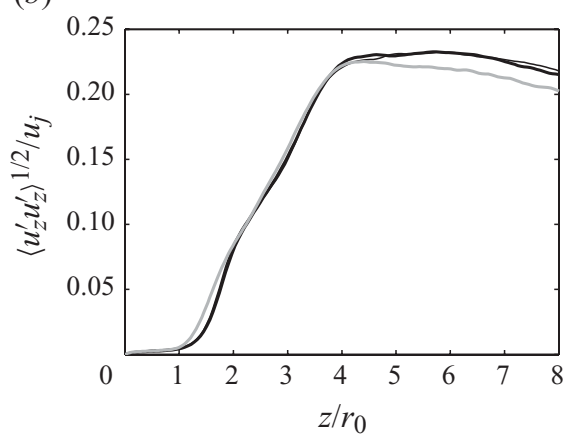

FIGURE 23. Variations of $(a)$ the shear-layer momentum thickness $\delta_{\theta}$ and $(b)$ the r.m.s. values of velocity $u_{z}^{\prime}$ at $r=r_{0}$, from:- JetD02, - JetD02Lz20 using the same grid shortened by $5 r_{0}$ in the axial direction, JetD02grid using a finer grid.

(a)

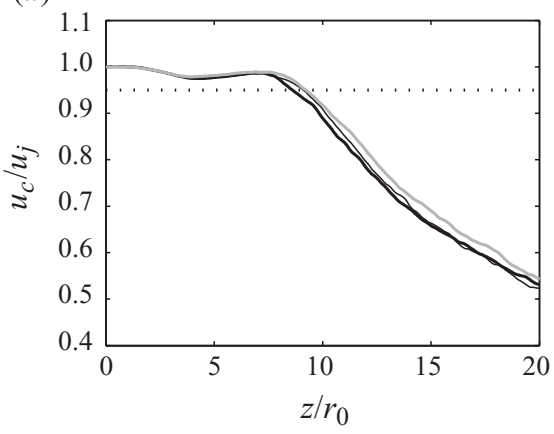

(b)

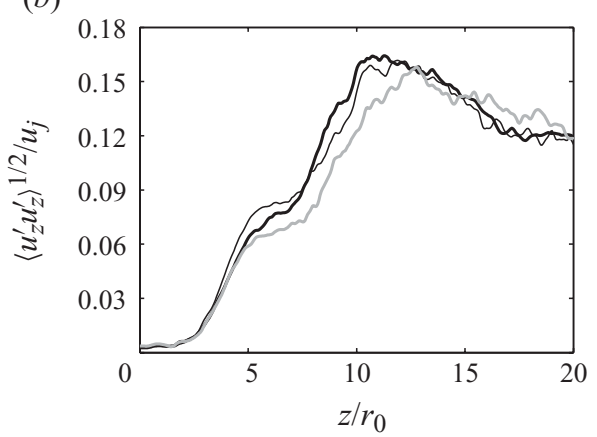

FIGURE 24. Variations of $(a)$ the centreline mean axial velocity $u_{c}$ and $(b)$ the r.m.s. values of the velocity $u_{z}^{\prime}$ along the centreline, from: grid shortened by $5 r_{0}$ in the axial direction, JetD02, JetD02Lz20 using the same JetD02grid using a finer grid.

take place slightly farther downstream with reduced turbulent intensities. The changes are however small in the latter case. The discrepancies between the results may also partially be due to a lack of statistical convergence. The aerodynamic properties of the initially laminar jets considered in this study can therefore reasonably be expected not to vary much with the sponge zone or with the grid resolution.

\section{Appendix C. Effects of grid parameters, control surface and inlet forcing on far-field noise}

In this appendix, the influence of the numerical parameters on far-field noise is addressed by reporting sound pressure spectra determined at $60 r_{0}$ from the pipe exit using the wave extrapolation method described in $\$ 2.4$.

In figure 25, sound spectra computed at the radiation angles of 40 and $90^{\circ}$ in different ways for an initially laminar jet with exit boundary layer of thickness $\delta=0.2 r_{0}$, from the simulations JetD02, JetD02Lz20 and JetD02grid defined in $\S 2.1$ and Appendix B, are shown. The spectra obtained using the full control surface located at $r=5.25 r_{0}$ for the far-field extrapolation are represented by solid lines. At 
(a)

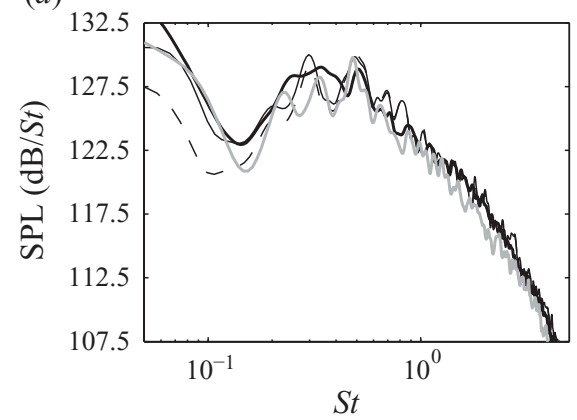

(b)

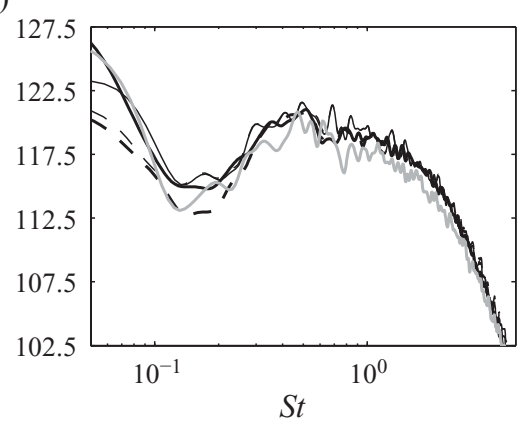

FIGURE 25. Narrow-band sound pressure levels (SPL) at $60 r_{0}$ from the jet exit for angles of (a) $40^{\circ}$ and $(b) 90^{\circ}$, as functions of $S t=f D / u_{j}$, from: - JetD02, JetD02Lz20, and - JetD02grid using the full control surface at $r=5.25 r_{0},-\quad-$ JetD02 using the surface at $r=5.25 r_{0}$ limited to $z=z_{c}+2 r_{0},--$ JetD02Lz20 using the full control surface at $r=7.25 r_{0}$.

both radiation angles considered, they exhibit very similar shapes and magnitudes, which supports that the far-field sound spectra in the present work do not significantly depend on the sponge zone or on the grid resolution of the LES. They also contain strong components for $S t \leqslant 0.1$.

To explore the origin of these low-frequency waves, visibly coming from the end of the control surface in figure 18, far-field extrapolations using control surfaces limited to $z=z_{c}+2 r_{0}$ in the axial direction, that is two radii downstream of the end of the potential core, have been carried out. The sound spectrum thus obtained at $90^{\circ}$ for JetD02 is shown in figure $25(b)$ by a thick dashed line. Compared with the calculations using the full control surface, the low-frequency noise components are of weaker amplitude whereas the high-frequency components are unchanged. A similar result is observed when a full control surface at $r=7.25 r_{0}$ is used instead of at $r=5.5 r_{0}$ for JetD02Lz20 (see the thin dashed line). The downstream part of the control surface at $r=5.5 r_{0}$ therefore appears too close to the aerodynamic flow field, which generates spurious waves for $S t \leqslant 0.1$, but fortunately does not affect the physical waves at higher Strouhal numbers.

It can moreover be pointed out that the spectra plotted in black in figure $25(a)$, computed at $40^{\circ}$ from JetD02 using a control surface at $r=5.25 r_{0}$ extending axially up to $z=25.5 r_{0}$, and from JetD02Lz20 using surfaces at $r=5.25 r_{0}$ or $r=7.25 r_{0}$ limited to $z=20.5 r_{0}$, do not differ significantly. The influence of the extrapolation surface on the sound spectra calculated in the downstream direction can therefore be expected to be relatively small.

Finally, a simulation JetD005p2000noflow has been performed using the numerical parameters and inlet random noise of JetD005p2000, but without flow to only characterize the noise radiated by the forcing. The sound spectrum calculated from this simulation in the acoustic far field at $90^{\circ}$ is plotted in figure 26, and compared with the corresponding spectrum from JetD005p2000. The noise generated by the forcing is at least $20 \mathrm{~dB}$ lower, all over the range of Strouhal numbers of interest. This demonstrates that the introduction of random disturbances in the pipe nozzle in JetD005p250 and JetD005p2000 has negligible impact on the acoustic far field. It can also be noted that the sound spectrum resulting from the inlet forcing does 


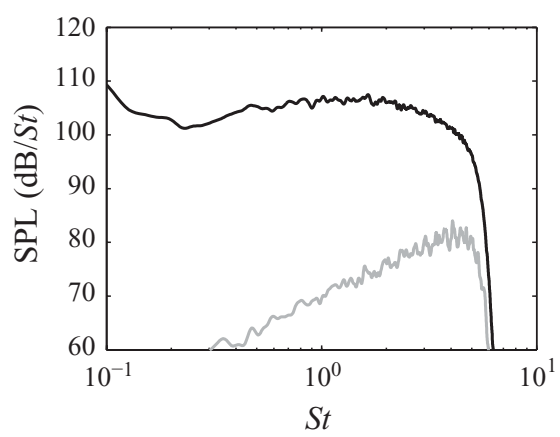

FIGURE 26. Narrow-band sound pressure levels (SPL) at $60 r_{0}$ from the jet exit for an angle of $90^{\circ}$, as functions of $S t=f D / u_{j}$, from: - JetD005p2000, JetD005p2000noflow using same grid and forcing but no jet flow.

not contain tone, which indicates that there is no acoustic resonance inside the nozzle.

\section{REFERENCES}

Ahuja, K. K., Tester, B. J. \& Tanna, H. K. 1987 Calculation of far field jet noise spectra from near field measurements with true source location. J. Sound Vib. 116 (3), 415-426.

Alkislar, M. B., Krothapalli, A. \& Butler, G. W. 2007 The effect of streamwise vortices on the aeroacoustics of a Mach 0.9. J. Fluid Mech. 578, 139-169.

Arakeri, V. H., Krothapalli, A., Siddavaram, V., Alkislar, M. B. \& Lourenco, L. 2003 On the use of microjets to suppress turbulence in a Mach 0.9 axisymmetric jet. J. Fluid Mech. 490, $75-98$.

Berland, J., Bogey, C. \& Bailly, C. 2007 a Numerical study of screech generation in a planar supersonic jet. Phys. Fluids 19, 075105.

Berland, J., Bogey, C., Marsden, O. \& Bailly, C. $2007 \mathrm{~b}$ High-order, low dispersive and low dissipative explicit schemes for multi-scale and boundary problems. J. Comput. Phys. 224 (2), $637-662$.

Bodony, D. J. \& Lele, S. K. 2008 On the current status of jet noise predictions using large-eddy simulation. AIAA J. 46 (2), 364-380.

Bogey, C. \& Bailly, C. 2002 Three-dimensional non-reflective boundary conditions for acoustic simulations: far-field formulation and validation test cases. Acta Acust. 88 (4), 463-471.

Bogey, C. \& Bailly, C. 2004 A family of low dispersive and low dissipative explicit schemes for flow and noise computations. J. Comput. Phys. 194 (1), 194-214.

Bogey, C. \& Bailly, C. 2005 Effects of inflow conditions and forcing on a Mach 0.9 jet and its radiated noise. AIAA J. 43 (5), 1000-1007.

Bogey, C. \& BaIlly, C. $2006 a$ Investigation of downstream and sideline subsonic jet noise using large-eddy simulations. Theoret. Comput. Fluid Dyn. 20 (1), 23-40.

Bogey, C. \& Bailly, C. $2006 b$ Large-eddy simulations of round jets using explicit filtering with/without dynamic Smagorinsky model. Intl J. Heat Fluid Flow 27 (4), 603-610.

Bogey, C. \& Bailly, C. 2006c Large-eddy simulations of transitional round jets: influence of the Reynolds number on flow development and energy dissipation. Phys. Fluids 18 (6), 065101.

Bogey, C. \& Bailly, C. 2007 An analysis of the correlations between the turbulent flow and the sound pressure field of subsonic jets. J. Fluid Mech. 583, 71-97.

Bogey, C. \& Bailly, C. 2009 Turbulence and energy budget in a self-preserving round jet: direct evaluation using large-eddy simulation. J. Fluid Mech. 627, 129-160.

Bogey, C., Barré, S. \& Bailly, C. 2008 Direct computation of the noise generated by subsonic jets originating from a straight pipe nozzle. Intl J. Aeroacoust. 7 (1), 1-22.

Bogey, C., Barré, S., Fleury, V., Bailly, C. \& Juvé, D. 2007 Experimental study of the spectral properties of near-field and far-field jet noise. Intl J. Aeroacoust. 6 (2), 73-92. 
Bogey, C., Barré, S., Juvé, D. \& Bailly, C. 2009a Simulation of a hot coaxial jet: direct noise prediction and flow-acoustics correlations. Phys. Fluids 21 (3), 035105.

Bogey, C., De Cacqueray, N. \& Bailly, C. $2009 \mathrm{~b}$ A shock-capturing methodology based on adaptative spatial filtering for high-order non-linear computations. J. Comput. Phys. 228 (5), $1447-1465$.

Bridges, J. E. \& Hussain, A. K. M. F. 1987 Roles of initial conditions and vortex pairing in jet noise. J. Sound Vib. 117 (2), 289-311.

Callender, B., Gutmark, E. \& Martens, S. 2005 Far-field acoustic investigation into chevron nozzle mechanisms and trends. AIAA J. 43 (1), 87-95.

Castelain, T., Sunyach, M., Juvé, D. \& BÉRA, J.-C. 2008 Jet-noise reduction by impinging microjets: an acoustic investigation testing microjet parameters. AIAA J. 46 (5), 1081-1087.

Cohen, J. \& Wygnanski, I. 1987 The evolution of instabilities in the axisymmetric jet. Part 1: The linear growth of disturbances near the nozzle. J. Fluid Mech. 176, 191-219.

Colonius, T. \& Lele, S. K. 2004 Computational aeroacoustics: progress on nonlinear problems of sound generation. Prog. Aerosp. Sci. 40, 345-416.

Crighton, D. G. 1981 Acoustics as a branch of fluid mechanics. J. Fluid Mech. 106, 261-298.

Fleury, V., Bailly, C., Jondeau, E., Michard, M. \& Juvé, D. 2008 Space-time correlations in two subsonic jets using dual-PIV measurements. AIAA J. 46 (10), 2498-2509.

Grosche, F.-R. 1974 Distributions of sound source intensities in subsonic and supersonic jets. AGARD-CP-131, 4-1 to 4-10. AGARD.

Hill, W. G., Jenkins, R. C. \& Gilbert, B. L. 1976 Effects of the initial boundary-layer state on turbulent jet mixing. AIAA J. 14 (11), 1513-1514.

Husain, Z. D. \& Hussain, A. K. M. F. 1979 Axisymmetric mixing layer: influence of the initial and boundary conditions. AIAA J. 17 (1), 48-55.

Hussain, A. K. M. F \& Zedan, M. F. 1978 a Effects of the initial condition on the axisymmetric free shear layer: effects of the initial momentum thickness. Phys. Fluids 21 (7), 1100-1112.

Hussain, A. K. M. F. \& Zedan, M. F. $1978 b$ Effects of the initial condition on the axisymmetric free shear layer: effects of the initial fluctuation level. Phys. Fluids 21 (9), 1475-1481.

Juvé, D. \& SunYaCH, M. 1978 Structure azimutale du champ acoustique lointain d'un jet subsonique. C. R. Acad. Sci. Paris 287 (B), 187-190.

Kearney-Fischer, M., Kim, J.-H. \& SAmimy, M. 2009 Control of a high Reynolds number Mach 0.9 heated jet using plasma actuators. Phys. Fluids 21 (9), 095101.

KIM, J. \& CHOI, H. 2009 Large eddy simulation of a circular jet: effect of inflow conditions on the near field. J. Fluid Mech. 620, 383-411.

LaU, J. C., Morris, P. J. \& Fisher, M. J. 1979 Measurements in subsonic and supersonic free jets using a laser velocimeter. J. Fluid Mech. 93 (1), 1-27.

LEPICOVSKY, J. \& BROWN, W. H. 1989 Effects of nozzle exit boundary-layer conditions on excitability of heated free jets. AIAA J. 27 (6), 712-718.

Lush, P. A. 1971 Measurements of subsonic jet noise and comparison with theory. J. Fluid Mech. 46 (3), 477-500.

Maestrello, L. 1976 Two points correlations of sound pressure in the far field of a jet: experiment. Tech. Memorandum 72835. NASA-Langley Research Center.

Maestrello, L. \& MCDAid, E. 1971 Acoustic characteristics of a high-subsonic jet. AIAA J. 9 (6), $1058-1066$.

Michalke, A. 1984 Survey on jet instability theory. Prog. Aerosp. Sci. 21, 159-199.

Mohseni, K. \& Colonius, T. 2000 Numerical treatment of polar coordinate singularities. J. Comput. Phys. 157 (2), 787-795.

Mollo-Christensen, E., Kolpin, M. A. \& Martucelli, J. R. 1964 Experiments on jet flows and jet noise far-field spectra and directivity patterns. J. Fluid Mech. 18, 285-301.

Morris, P. J. \& Zaman, K. B. M. Q. 2009 Velocity measurements in jets with application to noise source modelling. J. Sound Vib. 329 (4), 394-414.

Raman, G., Rice, E. J. \& Reshotko, E. 1994 Mode spectra of natural disturbances in a circular jet and the effect of acoustic forcing. Exp. Fluids 17, 415-426.

Raman, G., Zaman, K. B. M. Q. \& Rice, E. J. 1989 Initial turbulence effect on jet evolution with and without tonal excitation. Phys. Fluids A 1 (7), 1240-1248. 
Russ, S. \& STRYKowski, P. J. 1993 Turbulent structure and entrainment in heated jets: the effect of initial conditions. Phys. Fluids A 5 (12), 3216-3225.

Saiyed, N. H., Mikkelsen, K. L. \& Bridges, J. E. 2003 Acoustics and thrust of quiet separate-flow high-bypass-ratio nozzles. AIAA J. 41 (3), 372-378.

Stanley, S. A. \& Sarkar, S. 2000 Influence of nozzle conditions and discrete forcing on turbulent planar jets. AIAA J. 38 (9), 1615-1623.

Suzuki, T. \& Colonius, T. 2007 Instability waves in a subsonic round jet detected using a near-field phased microphone array. J. Fluid Mech. 565, 197-226.

TAM, C. K. W. \& Dong, Z. 1996 Radiation and outflow boundary conditions for direct computation of acoustic and flow disturbances in a nonuniform mean flow. J. Comput. Acoust. 4 (2), $175-201$.

Tam, C. K. W., Viswanathan, K., Ahuja, K. K. \& Panda, J. 2008 The sources of jet noise: experimental evidence. J. Fluid Mech. 615, 253-292.

Tanna, H. K. 1977 An experimental study of jet noise. Part I: Turbulent mixing noise. J. Sound Vib. 50 (3), 405-428.

Zaman, K. B. M. Q. 1985 a Effect of initial condition on subsonic jet noise. AIAA J. 23, 1370-1373.

Zaman, K. B. M. Q. $1985 b$ Far-field noise of subsonic jet under controlled excitation. J. Fluid Mech. 152, 83-111.

Zaman, K. B. M. Q. 1986 Flow field and near and far sound field of a subsonic jet. J. Sound Vib. $106(1), 1-16$.

Zaman, K. B. M. Q. \& Hussain, A. K. M. F. 1980 Vortex pairing in a circular jet under controlled excitation. Part 1. General jet response. J. Fluid Mech. 101 (3), 449-491. 